\title{
Oxygen isotopic composition of relict olivine grains in cosmic spherules: Links to chondrules from carbonaceous chondrites
}

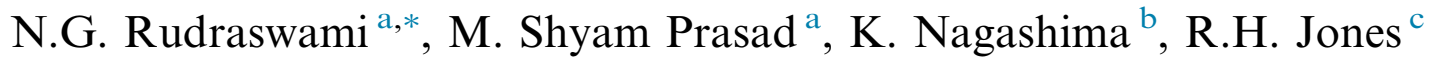 \\ ${ }^{a}$ National Institute of Oceanography (Council of Scientific and Industrial Research), Dona Paula, Goa 403004, India \\ ${ }^{\mathrm{b}}$ Hawaii Institute of Geophysics and Planetology, Hawai' $i$ at Māanoa, 1680 East-West-Road, Honolulu, HI 96822, USA \\ ${ }^{\mathrm{c}}$ Department of Earth and Planetary Sciences, University of New Mexico, Albuquerque, NM 87131, USA
}

Received 2 August 2014; accepted in revised form 4 May 2015; Available online 13 May 2015

\begin{abstract}
Most olivine relict grains in cosmic spherules selected for the present study are pristine and have not been disturbed during their atmospheric entry, thereby preserving their chemical, mineralogical and isotopic compositions. In order to understand the origin of the particles, oxygen isotope compositions of relict olivine grains in twelve cosmic spherules collected from deep sea sediments of the Indian Ocean were studied using secondary ion mass spectrometry. Most of the data lie close to the CCAM (Carbonaceous Chondrite Anhydrous Mineral) line, with $\Delta^{17} \mathrm{O}$ ranging from $-5 \%$ to $0 \%$. The data overlap oxygen isotopic compositions of chondrules from carbonaceous chondrites such as CV, CK, CR and CM, which suggests that chondrules from carbonaceous chondrites are the source of relict grains in cosmic spherules. Chemical compositions of olivine in cosmic spherules are also very similar to chondrule olivine from carbonaceous chondrites. Several olivine relict grains in three cosmic spherules are ${ }^{16} \mathrm{O}$-rich $\left(\Delta^{17} \mathrm{O}-21.9 \%\right.$ to $-18.7 \%$, similar to oxygen isotopic compositions observed in calcium aluminum rich inclusions (CAIs), amoeboid olivine aggregates (AOAs), and some porphyritic chondrules from carbonaceous chondrites. These grains appear to have recorded the initial oxygen isotopic composition of the inner solar nebula. Three olivine grains from two cosmic spherules have $\delta^{18} \mathrm{O}$ values $>+20 \%$, which could be interpreted as mixing with stratospheric oxygen during atmospheric entry.
\end{abstract}

(C) 2015 Elsevier Ltd. All rights reserved.

\section{INTRODUCTION}

Oxygen isotopes can play a significant role in unravelling the origin and processes undergone by small sized dust particles $(>10 \mu \mathrm{m}$ to $\sim$ few $\mathrm{mm})$ known as micrometeorites or cosmic spherules. These micrometeorites record evidence of formation processes in the early solar nebula if the materials are primitive. Others record evidence of evolved asteroids, similar to chondritic or achondritic parent bodies (e.g., Taylor et al., 2007; Genge et al., 2008; Gounelle

\footnotetext{
* Corresponding author.

E-mail address: rudra@nio.org (N.G. Rudraswami).
}

et al., 2009; Badjukov et al., 2010; Suavet et al., 2010; Cordier et al., 2011a,b, 2012; Van Ginneken et al., 2012; Cordier and Folco, 2014). Micrometeorites are a major source of extra-terrestrial dust particles with fluxes of $\sim 7500$ to 30,000 tonnes/yr (Love and Brownlee, 1993; Cremonese et al., 2012), although the material that survives Earth's atmospheric entry is much less (Taylor et al., 2000; Yada et al., 2004; Prasad et al., 2013). Micrometeorites are expected to have sampled a wide variety of parent bodies, including those known through meteorite studies, and some that have not yet been sampled (Brownlee, 1985; Gounelle et al., 2009; Badjukov et al., 2010). Understanding the origin of micrometeorites/cosmic spherules by comparing 
chemical compositions is not completely reliable for accurate parent body identification in view of the changes they undergo due to ablation during atmospheric entry (Cordier and Folco, 2014). In most cases it has been suggested on the basis of bulk chemistry that micrometeorites or cosmic spherules are related to carbonaceous chondrites (Kurat et al., 1994; Beckerling and Bischoff, 1995; Brownlee et al., 1997; Greshake et al., 1998; Cordier et al., 2011a; Rudraswami et al., 2012; Imae et al., 2013). However, there is no conclusive evidence on the relationship of the micrometeorites/cosmic spherules to the major components of meteorites such as chondrules and calcium-aluminum-rich inclusions (CAIs).

CAIs, the first solar system objects in the solar nebula, are formed by condensation of refractory minerals at high temperatures. They are ${ }^{16} \mathrm{O}$-rich, whereas chondrules, which are 1-2 Ma younger than CAIs, formed in relatively ${ }^{16} \mathrm{O}$-poor environments indicating a different set of environmental conditions during their formation (Clayton, 1993; Yurimoto et al., 1998; Jones et al., 2004; Rudraswami et al., 2011b; Nakashima et al., 2012; Ushikubo et al., 2012; Tenner et al., 2013, 2015). The oxygen isotope compositions of both chondrules and CAIs from carbonaceous chondrites fall close to the line defined as the Carbonaceous Chondrite Anhydrous Minerals (CCAM) line, which has a slope of $\sim 1$ on an oxygen three-isotope plot (Clayton et al., 1977, Clayton, 1993). High precision oxygen isotope studies on chondrules of the Acfer 094 ungrouped carbonaceous chondrite define a primitive chondrule minerals (PCM) line with slope $\sim 1$ that plots on the left side of the CCAM line (Ushikubo et al., 2012). Further high precision study on chondrules of different types of carbonaceous chondrites such as Yamato 81020 CO3.0 (Tenner et al., 2013), Meteorite Hills 00426 and Queen Alexandra Range 99177 CR3 (Tenner et al., 2015) confirms compositions that fall on the PCM line. Previous oxygen isotope studies done on individual minerals from Antarctica micrometeorites have a trend similar to the PCM line (Engrand et al., 1999; Gounelle et al., 2005). The oxygen isotopic composition of spinel in cosmic spherules is enriched in ${ }^{16} \mathrm{O}$ having $\delta^{17,18} \mathrm{O} \sim-40 \%$ similar to that of CAIs (Engrand et al., 1999; Taylor et al., 2012), while oxygen isotopic compositions of silicates are similar to those in chondrules found in carbonaceous chondrites (Engrand et al., 1999; Gounelle et al., 2009). The oxygen isotope compositions of melted cosmic spherules have extremely high $\delta^{18} \mathrm{O}$ suggesting that they undergo exchange with stratospheric oxygen during atmospheric entry (Clayton et al., 1986; Engrand et al., 2005). However, less melted or partially melted spherules have preserved isotopic composition suggesting these spherules represent chondrule fragments (Engrand et al., 1999, 2005).

In contrast to carbonaceous chondrites, bulk oxygen isotope compositions of chondrules in ordinary chondrites, as well as in-situ analyses of olivine and pyroxene in chondrules, fall above both the CCAM and the terrestrial fractionation (TF) lines (Clayton et al., 1983, 1991; Kita et al., 2010).

Here we report on the oxygen isotope compositions of relict grains in cosmic spherules that were recovered from the surficial sediments of the Indian Ocean. Relict grains are defined as grains that survived the heating of atmospheric entry: relict grains of olivine and rare pyroxene are usually $\mathrm{Mg}$-rich and have only minor Fe-rich zoning around their edges. More fayalite-rich olivine is also seen in some spherules: this has been described previously by Genge et al. (2008). Our selection of relict grains for analysis likely biases our observations to material derived from unequilibrated chondrite sources, in which forsterite is common, as opposed to equilibrated chondrites or differentiated meteorites. However, our objective is to address the origin of the relict grains, rather than to assess the source of the overall population of micrometeorites. Oxygen isotope compositions in the olivine grains that are analyzed here are compared with components of chondrites such as chondrules and CAIs. In addition, the oxygen isotope studies on cosmic spherules further improve our understanding of the stratospheric oxygen exchange expected during entry into Earth's atmosphere.

\section{SAMPLES AND EXPERIMENTAL PROCEDURES}

The cosmic spherules for the present study were collected from deep sea sediments of the central Indian Ocean, between longitude 74 to $76^{\circ} \mathrm{E}$ and latitude -13 to $-10^{\circ} \mathrm{S}$, at water depths of $\sim 5200 \mathrm{~m}$ with a grab sampler that penetrated the seafloor up to $15 \mathrm{~cm}$ (Rudraswami et al., 2011a, 2012; Prasad et al., 2013). Close to $\sim 3$ tonnes (wet weight) of sediment were sieved on two different cruises of Akademik Aleksandr Sidorenko (a research vessel chartered by the Government of India) of 30 days each, from 100 close-spaced sampling operations. The recovered sediments were sieved in a $\sim 200 \mu \mathrm{m}$ size mesh followed by magnetic separation. Cosmic spherules were handpicked from the magnetic separates under a binocular microscope, mounted in epoxy and polished to expose their internal features. Fifteen such mounts were prepared each containing more than a hundred spherules on a single section. For the present investigation, we selected cosmic spherules having potential relict grains with grain sizes $>20 \mu \mathrm{m}$. Efforts were made to select cosmic spherules that had at least two potential relict grains of the required size for ion microprobe analyses.

The polished sections were carbon coated and were observed using a scanning electron microscope (SEM JSM-5800LV at National Institute of Oceanography, Goa, India) for characterization, classification and to identify relict mineral grains greater than $20 \mu \mathrm{m}$ for oxygen isotope analyses using ion microprobe. Quantitative analyses of individual phases were obtained by an electron microprobe (Cameca SX5 at National Institute of Oceanography, Goa, India) using the following analytical parameters: accelerating voltage of $\sim 15 \mathrm{kV}$, beam current of $\sim 12 \mathrm{nA}$ and beam diameter of $\sim 1$ to $2 \mu \mathrm{m}$. All the mineral grains that were analyzed for oxygen isotopes were olivine, due to the large grain size available for the analyses. Multiple analyses were done on different mineral phases for each cosmic spherule and the bulk composition estimated using these compositions along with image analysis using the image $\mathbf{J}$ software program. Olivine is the dominant phase and there were no pyroxene phases in these cosmic 
spherules, however, some mesostasis was present in a few cosmic spherules. In addition, X-ray maps were obtained for different elements, using electron microprobe (wavelength dispersive spectrometers, WDS). Superimposing $\mathrm{Mg}, \mathrm{Fe}$, and $\mathrm{Ca}$ maps by assigning the colors red, green and blue, respectively, shows the presence of forsteritic relict grains, provides an understanding of elemental associations and helps to differentiate between the mineral phases.

In situ measurements of the oxygen isotope compositions of relict grains were done using the Cameca ims-1280 secondary ion mass spectrometer (SIMS or ion microprobe) at the University of Hawaii, Honolulu, USA. The $\mathrm{Cs}^{+}$primary beam with a current of $\sim 1 \mathrm{nA}$ rastered over $7 \times 7 \mu \mathrm{m}$ was used for measuring secondary oxygen ions. All isotopes of oxygen were measured simultaneously, ${ }^{16} \mathrm{O}^{-}$and ${ }^{18} \mathrm{O}^{-}$were detected using multi-collection Faraday cup (FC) detectors, and ${ }^{17} \mathrm{O}^{-}$was detected using monocollector electron multiplier. The mass resolving power set for ${ }^{16} \mathrm{O}^{-}$and ${ }^{18} \mathrm{O}^{-}$was $\sim 2000$, and that for ${ }^{17} \mathrm{O}^{-}$was $\sim 5600$ sufficient to resolve hydride interference ${ }^{16} \mathrm{OH}^{-}$. The contribution of interference ${ }^{16} \mathrm{OH}^{-}$on ${ }^{17} \mathrm{O}^{-}$ was verified after each analysis and was found to be insignificant $(<0.1 \%)$. Measured oxygen isotope ratios are expressed as $\delta^{17} \mathrm{O}$ and $\delta^{18} \mathrm{O}$, as permil deviation from Standard Mean Ocean Water (SMOW), expressed as $\left.\delta^{17,18} \mathrm{O}=\left[\left({ }^{17,18} \mathrm{O} /{ }^{16} \mathrm{O}\right)_{\text {sample }} /\left({ }^{17,18} \mathrm{O} /{ }^{16} \mathrm{O}\right)_{\text {SMOw }}-1\right) \times 1000\right]$. The deviation from the terrestrial mass fractionation line is expressed as $\Delta^{17} \mathrm{O}=\delta^{17} \mathrm{O}-0.52 \times \delta^{18} \mathrm{O}$ (Clayton, 1993). San Carlos olivine was used as a standard to correct instrumental mass fractionation for each session. External reproducibility of multiple standard analyses for both $\delta^{17} \mathrm{O}$ and $\delta^{18} \mathrm{O}$ was $\sim 0.5 \%$ to $1 \%(2 \sigma)$ and was propagated to errors of individual measurements. The individual analyzed spots were observed under SEM to identify cracks, grain borders and pits of ion microprobe analyses. All analyses done on cracks or grain boundaries were rejected.

\section{RESULTS}

\subsection{Petrography and chemistry}

Twelve cosmic spherules out of the $>1500$ observed contain relict grains $>20 \mu \mathrm{m}$ in size, indicating that the relict grains have survived atmospheric entry (Figs. 1 and 2). These relict grains are Mg-rich olivine having Fo $\geqslant 80 \%$, and are the best candidates to compare oxygen isotope compositions with other known extraterrestrial materials. The presence of forsterite-rich grains compared to the neighboring, more FeO-rich, olivine grains in cosmic spherules suggest the Fo-rich grains have not been affected by atmospheric heating and provide an opportunity to understand their original chemical and isotopic compositions. Three of the spherules, AAS38-43-P55, AAS62-61-P22 and AAS38-188-P43, are fine-grained and vesicular and are classified as relict bearing (Figs. 1 and $2 \mathrm{~b}, \mathrm{f}, \mathrm{g}$ ). The vesicular texture indicates escape of volatile elements, mostly likely as a result of heating during atmospheric entry. The other nine particles have porphyritic textures, similar to textures of porphyritic chondrules. AAS38-43-P38 and AAS62-51-P8 (Figs. 1 and 2a, h) both contain larger vesicles, located around the edges of the particles, which also probably indicates escape of volatile elements during atmospheric entry. The majority of the relict olivine grains have sizes in the range of $\sim 5$ to $30 \mu \mathrm{m}$; however, there are some that are quite large compared with the nominal range in grain size of spherules. AAS38-188-P43, AAS62-51-P8 and AAS62-40-P40 (Figs. 1 and 2g, h, j) contain large relict grains $\sim 100 \mu \mathrm{m}$ in size.

The approximate bulk chemical compositions of the cosmic spherules, and compositions of olivine grains where oxygen isotope analysis was done, are summarized in Tables 1 and 2, respectively. The olivine grains analyzed for oxygen isotopes are dominated by forsteritic compositions, excluding a few that are more FeO-rich, with Fo $\sim 60 \%$ (Table 2). Most olivine grains are zoned with a core having Fo $\sim 70 \%$ to $99 \%$ (Fig. 2). Combined X-ray elemental maps in $\mathrm{Mg}$ (red), $\mathrm{Fe}$ (green), $\mathrm{Ca}$ (blue) show that the cosmic spherules are dominated by forsteritic olivine (Fig. 2). There is also a small amount of mesostasis in many spherules; however, we have not analyzed mesostasis where mesostasis abundance is low and difficult to analyze by electron microprobe. In such cases mesostasis is not included in the bulk analyses. All the cosmic spherules are $\mathrm{Ca}$ - and $\mathrm{Al}$-poor (bulk $\mathrm{CaO}$ and bulk $\mathrm{Al}_{2} \mathrm{O}_{3}<4 \mathrm{wt} \%$ ) except AAS38-188-P15 which has a relatively high abundance of $\mathrm{Ca}$, Al-rich mesostasis (Fig. 2). Approximate bulk compositions for selected major elements have CI- and Si-normalized abundances close to 1, except for $\mathrm{Na}, \mathrm{Ca}$ and $\mathrm{Al}$ (Fig. 3). The $\mathrm{CaO}, \mathrm{MnO}$ and $\mathrm{Cr}_{2} \mathrm{O}_{3}$ concentration of the olivine grains analyzed for oxygen isotope studies fall within the ranges $0.1-0.6,0.1-0.4$ and $0.1-0.7 \mathrm{wt} \%$, respectively.

\subsection{Oxygen isotopes}

A total of 31 oxygen isotope analyses were made on olivine grains in 12 cosmic spherules (Table 3 ) and the oxygen three-isotope plots for individual cosmic spherules are shown in Fig. 4. The oxygen isotope compositions within each cosmic spherule are heterogeneous, but mostly lie close to the CCAM line. Values of $\delta^{18} \mathrm{O}, \delta^{17} \mathrm{O}$ and $\Delta^{17} \mathrm{O}$ of the analyzed olivine grains in these cosmic spherules range from $-39.7 \%$ to $30.9 \%,-41.8 \%$ to $9.2 \%$ and $-21.9 \%$ to $-2.2 \%$, respectively. Combined elemental $\mathrm{X}$-ray images marked with $\Delta^{17} \mathrm{O}$ values illustrate the spatial distribution of the analyzed spots (Fig. 2). AAS38-43-P38, AAS38-43-P55 and AAS62-61-P20 show a spread of compositions extending to low $\delta^{17,18} \mathrm{O}$ values, down to $\sim-40 \%$, similar to those seen in CAIs and some chondrules of carbonaceous chondrites (e.g., Clayton et al., 1977; Clayton, 1993; Yurimoto et al., 1998; McKeegan and Leshin, 2001; Wasson et al., 2001; Yurimoto and Wasson, 2002; Jones et al., 2004; Rudraswami et al., 2011b; Ushikubo et al., 2012). However, in AAS38-43-P55 and AAS62-61-P20 the spread in $\delta^{18} \mathrm{O}$ also extends to very high values, $>20 \%$, which lie close to the TF line. In situ ion microprobe measurements of the remainder of the cosmic spherules show oxygen isotope compositions similar to those in chondrule phenocrysts of carbonaceous chondrites, with $\delta^{18} \mathrm{O}$ values between $-10 \%$ and $+10 \%$ and plotting along the slope $\sim 1$ line. 

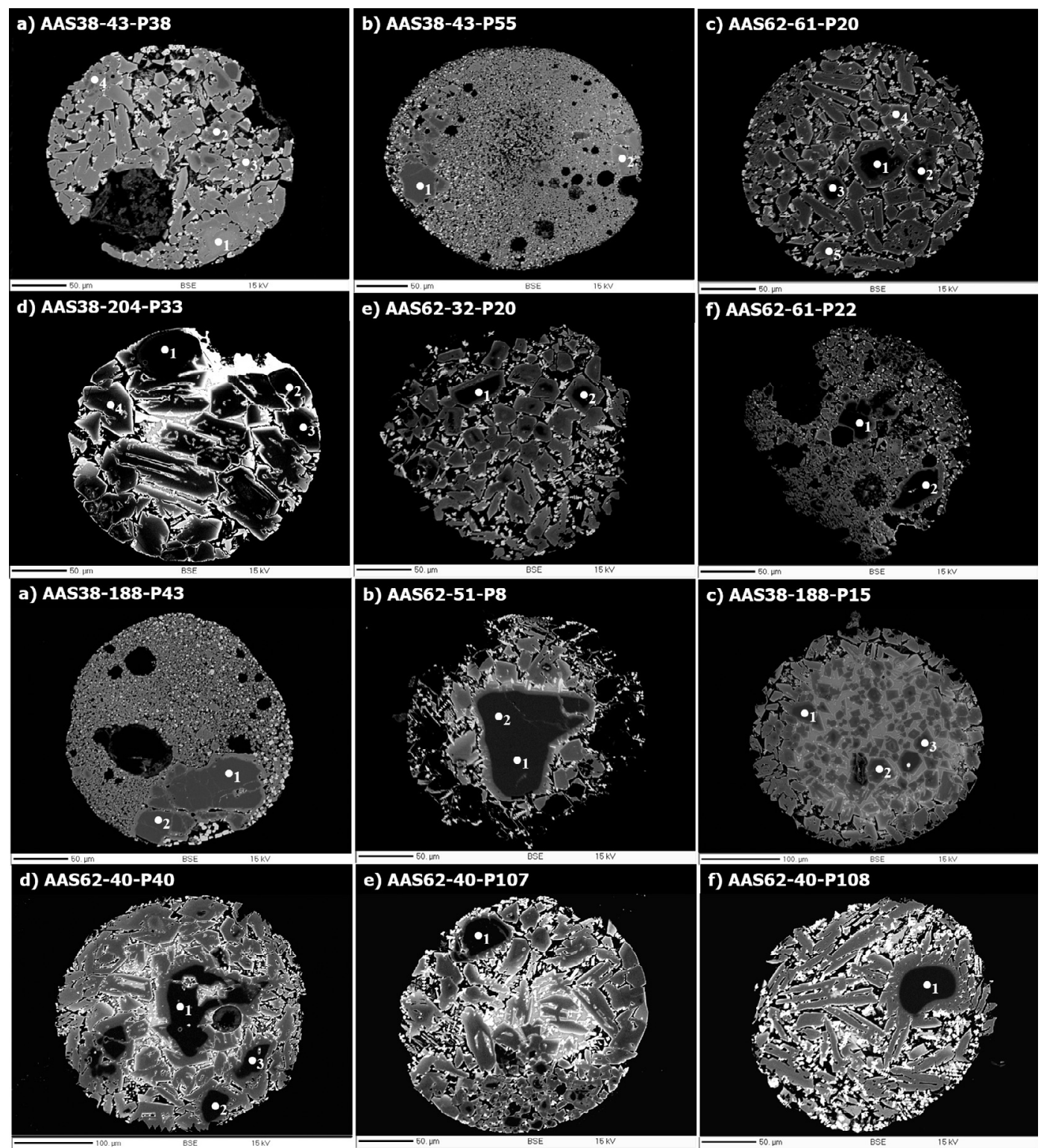

Fig. 1. Backscattered electron (BSE) images of cosmic spherules showing the presence of relict olivine grains. Forsterite-rich grains can be clearly distinguished because they are dark gray. Numbers indicate the locations of chemical analyses given in Table 2 and oxygen isotope analyses given in Table 3. Most of the olivine grains selected for oxygen isotope analyses are $>20 \mu \mathrm{m}$ in size. Cosmic spherules AAS38-43-P55, AAS62-61-P22, AAS38-188-P43 have fine-grained, vesicular, relict-bearing textures, while AAS38-43-P38, AAS62-61-P20, AAS38-204-P33, AAS62-32-P20, AAS62-51-P8, AAS38-188-P15, AAS62-40-P40, AAS62-40-P107, AAS62-40-P108 have porphyritic textures.

\section{DISCUSSION}

\subsection{Link to chondrules in carbonaceous chondrites}

Although chondrules are the major components of chondrites (up to $80 \%$ ), the contribution of chondrules to the micrometeorite flux remains uncertain. In addition to oxygen isotope evidence, discussed below, several of the cosmic spherules in this study appear to have porphyritic igneous textures, similar to chondrules, which suggests that some individual particles could actually be represent chondrules or fragments of chondrules that have survived as interplanetary dust particles following collisions on asteroids. Sizes of the micrometeorites, 100-200 $\mu \mathrm{m}$, are comparable to the average sizes of chondrules in $\mathrm{CO}$ and $\mathrm{CM}$ chondrites $(\sim 150$ and $300 \mu \mathrm{m}$, respectively, e.g., Jones, 2012). In addition, the oxygen isotopic compositions of olivine grains in $\mathrm{CO}$ and $\mathrm{CM}$ chondrites are similar to those found in the present study (Hiyagon and Hashimoto, 1999; Jones et al., 2000). It should also be noted that the ${ }^{16} \mathrm{O}$ enriched phases of cosmic spherules could also be related to AOAs (amoeboid olivine aggregates) which have similar oxygen isotopic composition (Imai and Yurimoto, 2003; Fagan et al., 2004; Krot et al., 2004a,b). The survival of a particle during atmospheric entry mainly depends on the zenith angle and velocity of 

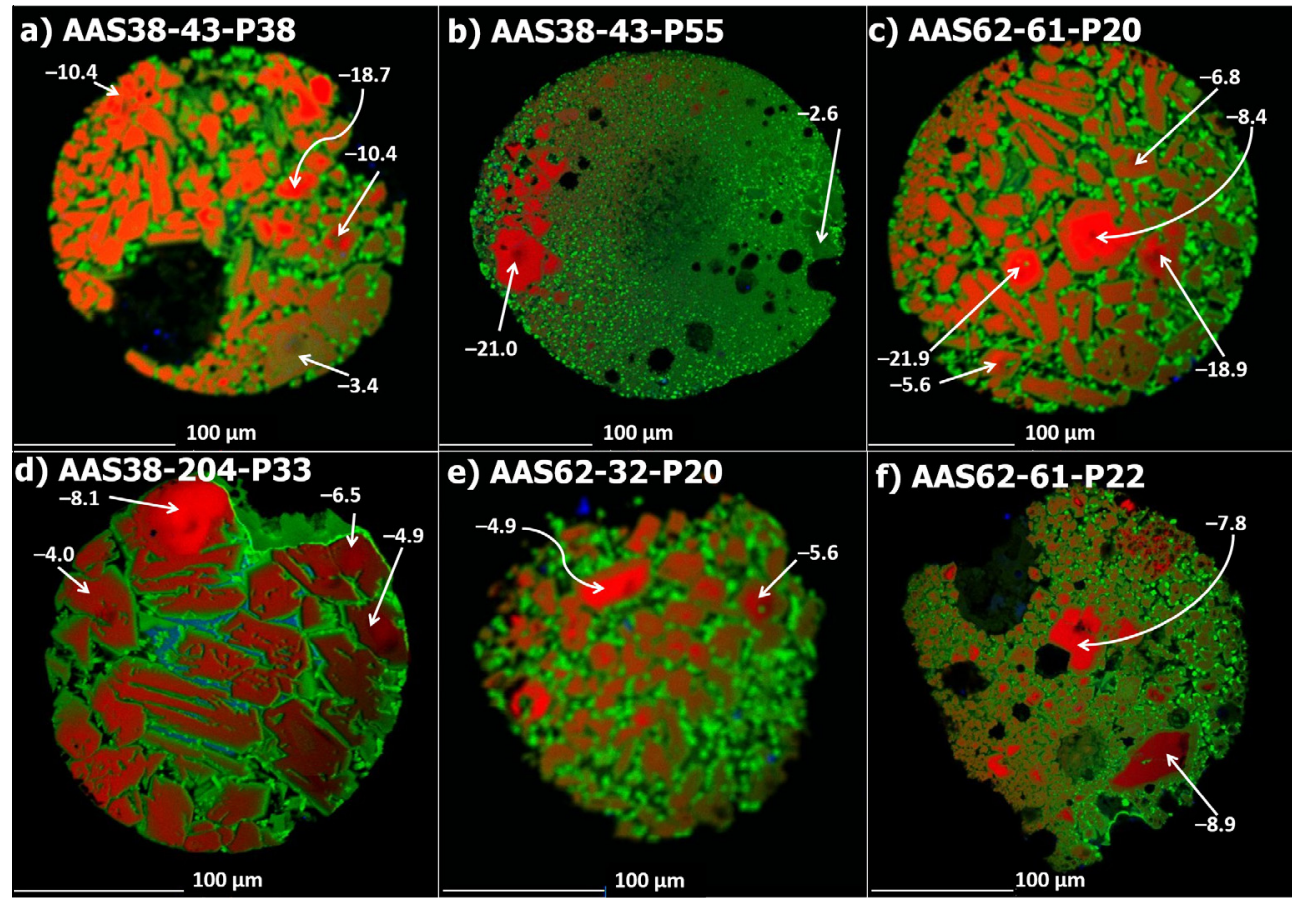

e) AAS62-32-P20
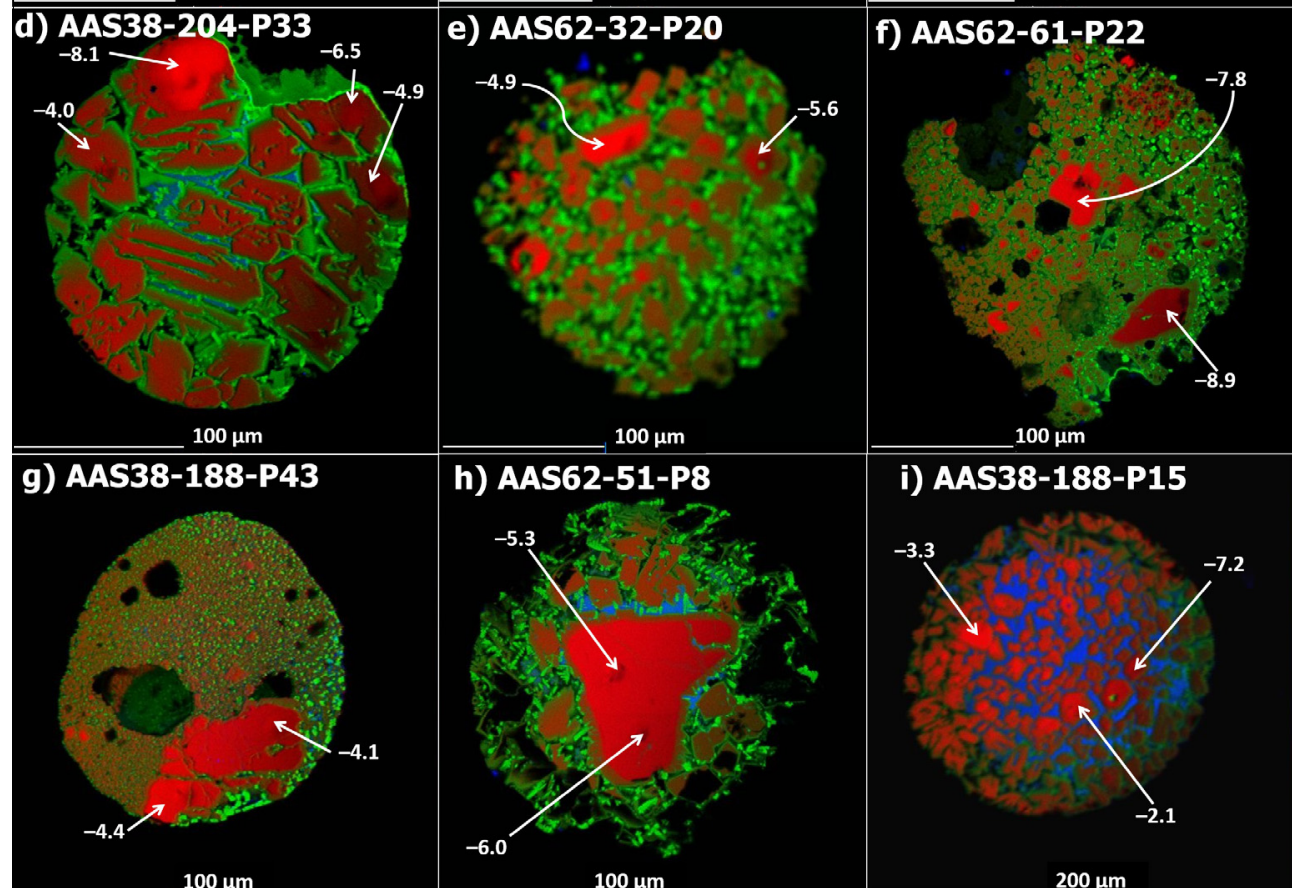

h) AAS62-51-P8
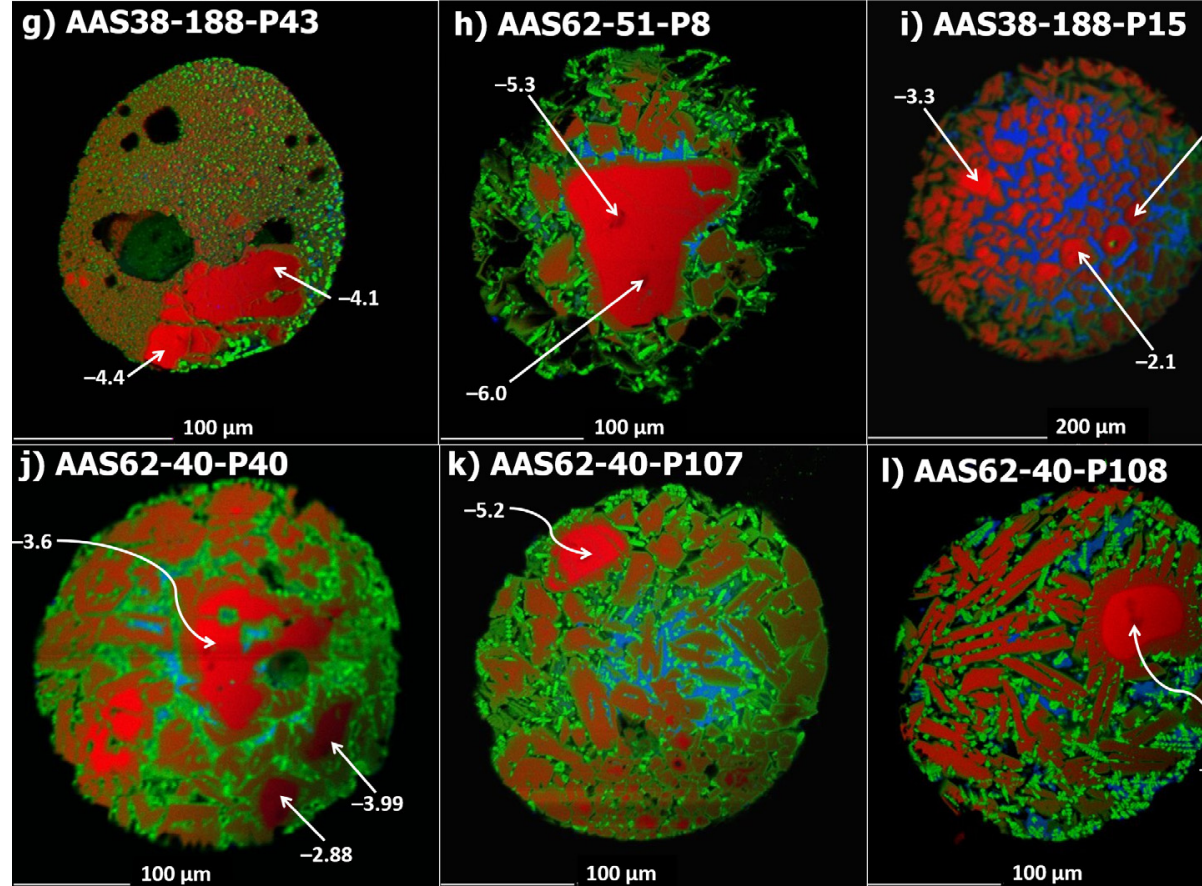

k) AAS62-40-P107
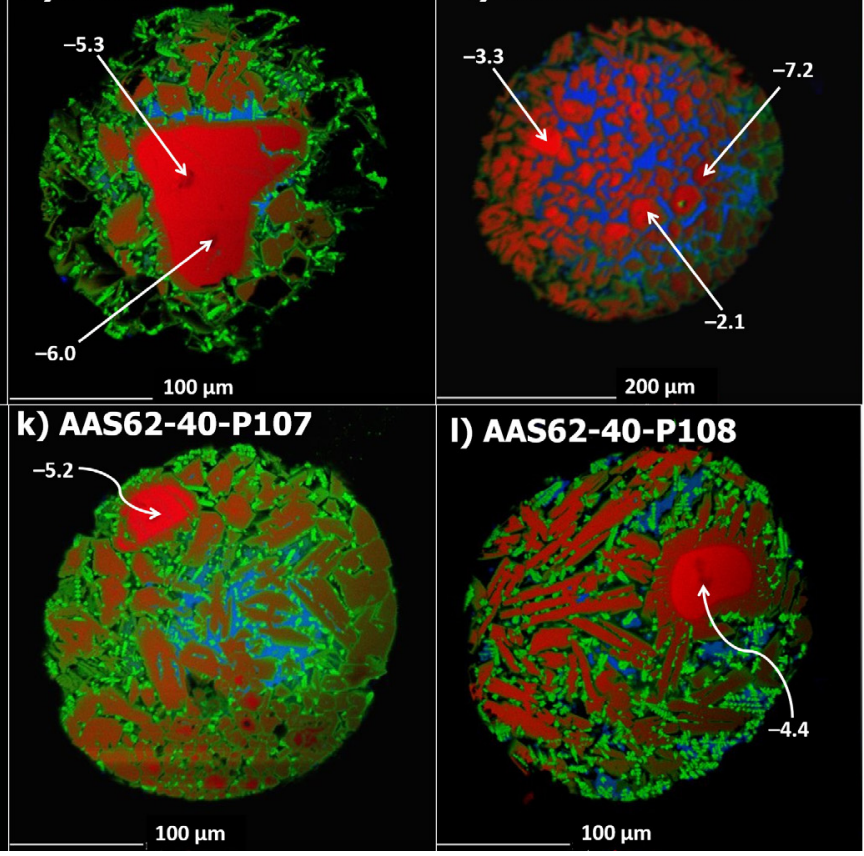

Fig. 2. Combined X-ray elemental maps of $\mathrm{Mg}$ (red), $\mathrm{Fe}$ (green) and $\mathrm{Ca}$ (blue) for the cosmic spherules analyzed for oxygen isotopes. Values of $\Delta^{17} \mathrm{O}$ are indicated in white. The bright red color indicates the olivine grains that have high Fo contents. Several of the analyzed cosmic spherules have Ca-rich mesostasis (blue), particularly AAS38-188-P15. (For interpretation of the references to color in this figure legend, the reader is referred to the web version of this article.)

the particle. Large zenith angle and lower velocity during entry will preserve the particle, although the percentage with these orbital parameters is small (Love and Brownlee, 1993). The large (up to $100 \mu \mathrm{m}$ ) crystal sizes of olivine grains, presence of zoning, presence of $\mathrm{Ca}$, Al-rich mesostasis, and the chemistry of the cosmic spherules selected in the present study bear a strong resemblance to porphyritic olivine (PO) chondrules (Genge et al., 2005). Chondrule textures comparable to those in Figs. 1 and 2 are illustrated in many papers on chondrules (e.g., Jones, 2012). Type II chondrules, which are FeO-rich, contain zoned olivine grains that commonly have forsteritic cores (e.g., Jones, 1992; Berlin et al., 2011). The forsteritic cores are interpreted as relict grains in the sense that they 
Table 1

Bulk chemical composition ( $\mathrm{wt} \%$ ) of cosmic spherules analyzed for oxygen isotope composition.

\begin{tabular}{|c|c|c|c|c|c|c|c|c|c|c|c|c|c|}
\hline Cosmic spherule & Type & $n^{\mathrm{a}}$ & $\mathrm{Na}_{2} \mathrm{O}$ & $\mathrm{MgO}$ & $\mathrm{Al}_{2} \mathrm{O}_{3}$ & $\mathrm{SiO}_{2}$ & $\mathrm{CaO}$ & $\mathrm{TiO}_{2}$ & $\mathrm{Cr}_{2} \mathrm{O}_{3}$ & $\mathrm{MnO}$ & $\mathrm{FeO}$ & $\mathrm{NiO}$ & Total \\
\hline AAS38-43-P38 & Porphyritic & 15 & 0.1 & 33.2 & 0.4 & 36.2 & 0.3 & - & 0.4 & 0.3 & 28.1 & 0.4 & 99.5 \\
\hline AAS38-43-P55 & Relict bearing & 9 & 0.1 & 33.2 & 0.4 & 36.2 & 0.3 & - & 0.4 & 0.3 & 28.1 & 0.4 & 99.5 \\
\hline AAS62-61-P20 & Porphyritic & 13 & - & 41.3 & 0.2 & 38.3 & 0.2 & - & 0.3 & 0.2 & 17.3 & 0.7 & 98.6 \\
\hline AAS38-204-P33 & Porphyritic & 18 & 0.1 & 31.6 & 3.0 & 38.8 & 2.6 & 0.1 & 0.2 & 0.2 & 24.2 & 0.1 & 101.4 \\
\hline AAS62-32-P20 & Porphyritic & 8 & - & 41.7 & 0.1 & 38.2 & 0.2 & - & 0.3 & 0.2 & 17.4 & 2.6 & 101.0 \\
\hline AAS62-61-P22 & Relict bearing & 8 & 0.1 & 33.2 & 1.8 & 36.6 & 0.3 & - & 0.3 & 0.2 & 26.8 & 0.2 & 99.7 \\
\hline AAS38-188-P43 & Relict bearing & 15 & - & 47.4 & 0.2 & 40.0 & 0.4 & 0.1 & 0.4 & 0.1 & 11.8 & 0.4 & 100.7 \\
\hline AAS62-51-P8 & Porphyritic & 11 & - & 44.9 & 0.2 & 39.9 & 0.3 & - & 0.3 & 0.1 & 13.0 & 0.7 & 99.5 \\
\hline AAS38-188-P15 & Porphyritic & 21 & - & 23.6 & 3.2 & 38.5 & 6.0 & 0.1 & 0.3 & 0.3 & 27.3 & 0.0 & 99.8 \\
\hline AAS62-40-P40 & Porphyritic & 17 & - & 45.1 & 0.2 & 39.0 & 0.2 & - & 0.3 & 0.1 & 11.3 & 0.4 & 96.8 \\
\hline AAS62-40-P107 & Porphyritic & 22 & - & 36.0 & 1.2 & 37.6 & 0.7 & - & 0.3 & 0.2 & 21.4 & 0.5 & 98.3 \\
\hline AAS62-40-P108 & Porphyritic & 10 & - & 30.3 & 1.5 & 39.4 & 3.8 & 0.1 & 0.2 & 0.3 & 20.6 & 0.8 & 97.1 \\
\hline
\end{tabular}

${ }^{a}$ Numbers of spot analysis used for obtaining the average.

Table 2

Chemical composition ( $\mathrm{wt} \%$ ) of relict olivine analyzed for oxygen isotope composition.

\begin{tabular}{|c|c|c|c|c|c|c|c|c|c|c|c|c|c|c|}
\hline Cosmic spherule & Type & $\#$ & $\mathrm{Na}_{2} \mathrm{O}$ & $\mathrm{MgO}$ & $\mathrm{Al}_{2} \mathrm{O}_{3}$ & $\mathrm{SiO}_{2}$ & $\mathrm{CaO}$ & $\mathrm{TiO}_{2}$ & $\mathrm{Cr}_{2} \mathrm{O}_{3}$ & $\mathrm{MnO}$ & $\mathrm{FeO}$ & $\mathrm{NiO}$ & Total & $\mathrm{Fo} \%$ \\
\hline AAS38-43-P38 & Porphyritic & $\begin{array}{l}1 \\
2 \\
3 \\
4\end{array}$ & $\begin{array}{l}0.1 \\
0.3 \\
0.1 \\
-\end{array}$ & $\begin{array}{l}28.9 \\
46.7 \\
29.4 \\
35.3\end{array}$ & $\begin{array}{l}- \\
0.2 \\
1.1 \\
0.5\end{array}$ & $\begin{array}{l}36.0 \\
38.7 \\
31.9 \\
36.4\end{array}$ & $\begin{array}{l}0.4 \\
0.2 \\
0.3 \\
0.2\end{array}$ & $\begin{array}{l}- \\
- \\
0.1 \\
-\end{array}$ & $\begin{array}{l}0.2 \\
0.2 \\
0.7 \\
0.4\end{array}$ & $\begin{array}{l}0.4 \\
0.1 \\
0.2 \\
0.3\end{array}$ & $\begin{array}{l}33.4 \\
12.1 \\
35.2 \\
26.5\end{array}$ & $\begin{array}{l}0.1 \\
0.5 \\
0.6 \\
0.3\end{array}$ & $\begin{array}{l}99.4 \\
99.0 \\
99.6 \\
99.9\end{array}$ & $\begin{array}{l}60.7 \\
87.3 \\
59.8 \\
70.4\end{array}$ \\
\hline AAS38-43-P55 & Relict bearing & $\begin{array}{l}1 \\
2\end{array}$ & $\begin{array}{l}0.1 \\
0.2\end{array}$ & $\begin{array}{l}53.5 \\
32.9\end{array}$ & $\begin{array}{l}- \\
0.3\end{array}$ & $\begin{array}{l}42.3 \\
36.2\end{array}$ & $\begin{array}{l}0.2 \\
0.3\end{array}$ & $\begin{array}{l}- \\
0.1\end{array}$ & $\begin{array}{l}0.4 \\
0.7\end{array}$ & $\begin{array}{l}0.2 \\
0.2\end{array}$ & $\begin{array}{r}3.9 \\
28.2\end{array}$ & $\begin{array}{l}0.1 \\
0.6\end{array}$ & $\begin{array}{r}100.6 \\
99.6\end{array}$ & $\begin{array}{l}96.1 \\
67.5\end{array}$ \\
\hline AAS62-61-P20 & Porphyritic & $\begin{array}{l}1 \\
2 \\
3 \\
4 \\
5\end{array}$ & $\begin{array}{l}- \\
- \\
- \\
- \\
0.1\end{array}$ & $\begin{array}{l}54.6 \\
48.3 \\
53.5 \\
38.2 \\
40.6\end{array}$ & $\begin{array}{l}- \\
0.3 \\
0.6 \\
0.2 \\
0.1\end{array}$ & $\begin{array}{l}41.7 \\
40.4 \\
41.4 \\
37.8 \\
37.9\end{array}$ & $\begin{array}{l}0.2 \\
0.3 \\
0.3 \\
0.2 \\
0.1\end{array}$ & $\begin{array}{l}- \\
- \\
- \\
- \\
-\end{array}$ & $\begin{array}{l}0.5 \\
0.4 \\
0.5 \\
0.3 \\
0.2\end{array}$ & $\begin{array}{l}0.2 \\
0.2 \\
0.2 \\
0.2 \\
0.1\end{array}$ & $\begin{array}{r}1.4 \\
9.7 \\
2.7 \\
21.1 \\
17.6\end{array}$ & $\begin{array}{l}0.1 \\
0.2 \\
- \\
1.3 \\
0.7\end{array}$ & $\begin{array}{l}98.6 \\
99.9 \\
99.2 \\
99.4 \\
97.6\end{array}$ & $\begin{array}{l}98.6 \\
89.9 \\
97.3 \\
76.4 \\
80.4\end{array}$ \\
\hline AAS38-204-P33 & Porphyritic & $\begin{array}{l}1 \\
2 \\
3 \\
4\end{array}$ & $\begin{array}{l}- \\
- \\
- \\
0.1\end{array}$ & $\begin{array}{l}51.1 \\
48.8 \\
44.5 \\
38.5\end{array}$ & $\begin{array}{l}0.2 \\
0.2 \\
0.2 \\
0.2\end{array}$ & $\begin{array}{l}41.5 \\
41.2 \\
40.5 \\
37.9\end{array}$ & $\begin{array}{l}0.5 \\
0.5 \\
0.6 \\
0.1\end{array}$ & $\begin{array}{l}0.1 \\
0.1 \\
0.1 \\
-\end{array}$ & $\begin{array}{l}0.3 \\
0.2 \\
0.3 \\
0.4\end{array}$ & $\begin{array}{l}- \\
0.1 \\
0.2 \\
0.3\end{array}$ & $\begin{array}{r}5.4 \\
10.3 \\
13.8 \\
22.5\end{array}$ & $\begin{array}{l}- \\
0.1 \\
- \\
0.1\end{array}$ & $\begin{array}{r}99.2 \\
101.5 \\
100.1 \\
100.3\end{array}$ & $\begin{array}{l}94.4 \\
89.4 \\
85.2 \\
75.3\end{array}$ \\
\hline AAS62-32-P20 & Porphyritic & $\begin{array}{l}1 \\
2\end{array}$ & - & $\begin{array}{l}53.4 \\
52.4\end{array}$ & $\begin{array}{l}- \\
-\end{array}$ & $\begin{array}{l}40.6 \\
40.4\end{array}$ & $\begin{array}{l}0.2 \\
0.2\end{array}$ & $\begin{array}{l}- \\
-\end{array}$ & $\begin{array}{l}0.4 \\
0.3\end{array}$ & $\begin{array}{l}0.1 \\
0.2\end{array}$ & $\begin{array}{l}2.5 \\
6.2\end{array}$ & $\begin{array}{l}0.1 \\
0.2\end{array}$ & $\begin{array}{l}97.3 \\
99.9\end{array}$ & $\begin{array}{l}97.4 \\
93.8\end{array}$ \\
\hline AAS62-61-P22 & Relict bearing & $\begin{array}{l}1 \\
2\end{array}$ & $\begin{array}{l}- \\
-\end{array}$ & $\begin{array}{l}54.5 \\
53.9\end{array}$ & $\begin{array}{l}- \\
0.1\end{array}$ & $\begin{array}{l}42.0 \\
41.5\end{array}$ & $\begin{array}{l}0.2 \\
0.2\end{array}$ & $\begin{array}{l}0.1 \\
-\end{array}$ & $\begin{array}{l}0.5 \\
0.3\end{array}$ & $\begin{array}{l}0.1 \\
0.2\end{array}$ & $\begin{array}{l}1.3 \\
1.3\end{array}$ & $\begin{array}{l}- \\
-\end{array}$ & $\begin{array}{l}98.7 \\
97.4\end{array}$ & $\begin{array}{l}98.6 \\
98.7\end{array}$ \\
\hline AAS38-188-P43 & Relict bearing & $\begin{array}{l}1 \\
2\end{array}$ & $\begin{array}{l}- \\
-\end{array}$ & $\begin{array}{l}55.8 \\
56.1\end{array}$ & $\begin{array}{l}0.2 \\
0.2\end{array}$ & $\begin{array}{l}42.4 \\
42.0\end{array}$ & $\begin{array}{l}0.4 \\
0.4\end{array}$ & $\begin{array}{l}0.1 \\
0.1\end{array}$ & $\begin{array}{l}0.5 \\
0.4\end{array}$ & $\begin{array}{l}0.1 \\
0.1\end{array}$ & $\begin{array}{l}1.2 \\
1.1\end{array}$ & $\begin{array}{l}- \\
0.1\end{array}$ & $\begin{array}{l}100.7 \\
100.5\end{array}$ & $\begin{array}{l}98.8 \\
98.9\end{array}$ \\
\hline AAS62-51-P8 & Porphyritic & $\begin{array}{l}1 \\
2\end{array}$ & $\begin{array}{l}0.1 \\
-\end{array}$ & $\begin{array}{l}54.2 \\
55.4\end{array}$ & $\begin{array}{l}0.2 \\
0.2\end{array}$ & $\begin{array}{l}42.3 \\
42.6\end{array}$ & $\begin{array}{l}0.4 \\
0.5\end{array}$ & $\begin{array}{l}0.1 \\
-\end{array}$ & $\begin{array}{l}0.2 \\
0.2\end{array}$ & - & $\begin{array}{l}1.3 \\
0.9\end{array}$ & $\begin{array}{l}- \\
-\end{array}$ & $\begin{array}{l}98.9 \\
99.8\end{array}$ & $\begin{array}{l}98.7 \\
99.1\end{array}$ \\
\hline AAS38-188-P15 & Porphyritic & $\begin{array}{l}1 \\
2 \\
3\end{array}$ & $\begin{array}{l}- \\
0.1 \\
-\end{array}$ & $\begin{array}{l}55.2 \\
51.5 \\
44.1\end{array}$ & $\begin{array}{l}- \\
0.1 \\
0.1\end{array}$ & $\begin{array}{l}43.8 \\
41.7 \\
39.7\end{array}$ & $\begin{array}{l}0.2 \\
0.2 \\
0.3\end{array}$ & $\begin{array}{l}- \\
- \\
-\end{array}$ & $\begin{array}{l}0.5 \\
0.7 \\
0.4\end{array}$ & $\begin{array}{l}0.1 \\
0.3 \\
0.2\end{array}$ & $\begin{array}{r}2.0 \\
5.7 \\
15.8\end{array}$ & $\begin{array}{l}- \\
- \\
0.1\end{array}$ & $\begin{array}{l}101.9 \\
100.2 \\
100.7\end{array}$ & $\begin{array}{l}98.0 \\
94.2 \\
83.2\end{array}$ \\
\hline AAS62-40-P40 & Porphyritic & $\begin{array}{l}1 \\
2 \\
3\end{array}$ & $\begin{array}{l}- \\
- \\
-\end{array}$ & $\begin{array}{l}55.4 \\
55.5 \\
55.6\end{array}$ & $\begin{array}{l}0.1 \\
0.1 \\
0.2\end{array}$ & $\begin{array}{l}42.2 \\
42.6 \\
42.2\end{array}$ & $\begin{array}{l}0.3 \\
0.3 \\
0.3\end{array}$ & $\begin{array}{l}0.1 \\
0.1 \\
-\end{array}$ & $\begin{array}{l}0.2 \\
0.1 \\
0.2\end{array}$ & $\begin{array}{l}- \\
- \\
0.1\end{array}$ & $\begin{array}{l}0.6 \\
0.7 \\
1.2\end{array}$ & $\begin{array}{l}- \\
- \\
-\end{array}$ & $\begin{array}{l}98.9 \\
99.4 \\
99.7\end{array}$ & $\begin{array}{l}99.4 \\
99.3 \\
98.8\end{array}$ \\
\hline AAS62-40-P107 & Porphyritic & 1 & - & 53.8 & 0.3 & 41.1 & 0.5 & 0.1 & 0.1 & - & 1.7 & 0.1 & 97.7 & 98.2 \\
\hline AAS62-40-P108 & Porphyritic & 1 & - & 54.8 & 0.1 & 42.4 & 0.3 & - & 0.5 & 0.1 & 1.1 & - & 99.2 & 98.9 \\
\hline
\end{tabular}

survived chondrule formation. Similar textures are observed in cosmic spherules AAS38-43-P38, AAS62-61-P20, AAS62-32-P20, AAS62-51-P8, AAS62-40-P40, AAS62-40-P107, and AAS62-40-P108.
Relict olivine grains of cosmic spherules have $\mathrm{CaO}$, $\mathrm{MnO}$, and $\mathrm{Cr}_{2} \mathrm{O}_{3}$ contents between $0.1-0.6,0.1-0.4$ and $0.1-0.7 \mathrm{wt} \%$ (Table 2), within the range of compositions of chondrule olivine from carbonaceous and ordinary 


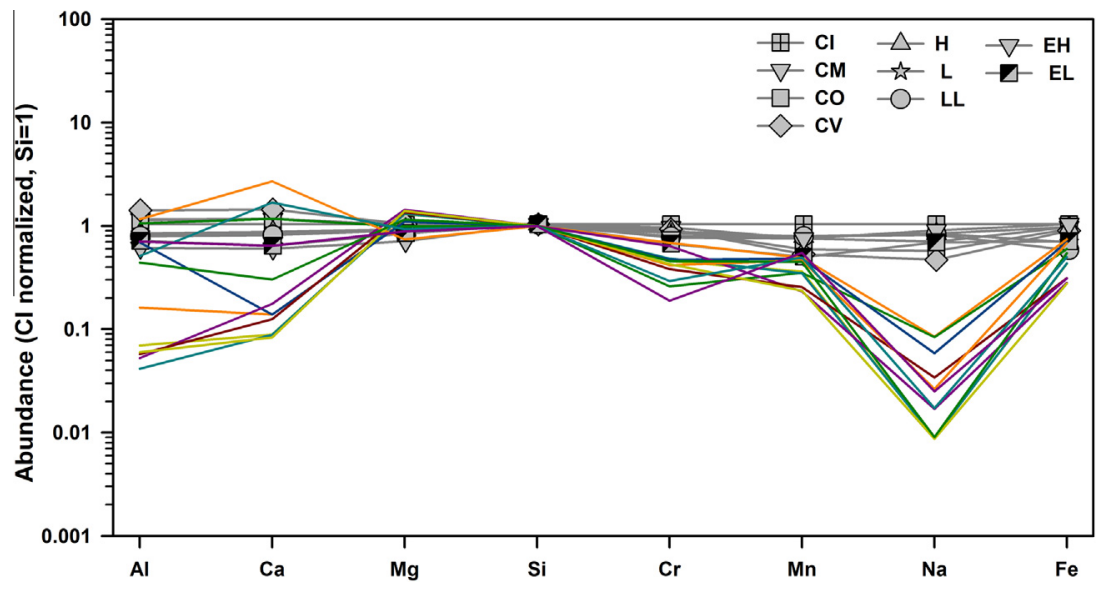

Fig. 3. CI and Si normalized abundances of major elements in cosmic spherules, compared with bulk analyses of different types of chondrites. The gray lines are chondrite bulk compositions (Lodders and Fegley, 1998). The majority of the elements in cosmic spherules are close to CI value except in the case of $\mathrm{Na}$, while $\mathrm{Al}$ and $\mathrm{Ca}$ are also $<1 \times \mathrm{CI}$ in many cases. AAS38-188-P15 is the only spherule that has $\mathrm{Ca}>1$ (orange line). (For interpretation of the references to color in this figure legend, the reader is referred to the web version of this article.)

Table 3

Oxygen isotopic composition of relict olivine grains in cosmic spherules. The oxygen isotope $\delta$ values are in \% .

\begin{tabular}{|c|c|c|c|c|c|c|c|c|c|}
\hline Cosmic spherule & Type & $\#$ & $\mathrm{Fo}^{2} \%$ & $\delta^{18} \mathrm{O}$ & $2 \sigma$ & $\delta^{17} \mathrm{O}$ & $2 \sigma$ & $\Delta^{17} \mathrm{O}$ & $\overline{2 \sigma}$ \\
\hline \multirow[t]{4}{*}{ AAS-38-43-P38 } & Porphyritic & 1 & 60.7 & 1.2 & 0.7 & -2.8 & 0.7 & -3.5 & 0.8 \\
\hline & & 2 & 87.3 & -30.6 & 0.7 & -34.7 & 0.6 & -18.8 & 0.8 \\
\hline & & 3 & 59.8 & -9.7 & 0.9 & -16.1 & 0.7 & -11.0 & 0.9 \\
\hline & & 4 & 70.4 & -8.4 & 1.0 & -14.8 & 0.9 & -10.5 & 1.1 \\
\hline \multirow[t]{2}{*}{ AAS-38-43-P55 } & Relict bearing & 1 & 96.1 & -39.7 & 0.7 & -41.8 & 0.6 & -21.1 & 0.7 \\
\hline & & 2 & 67.5 & 20.5 & 0.7 & 7.9 & 0.6 & -2.7 & 0.7 \\
\hline \multirow[t]{5}{*}{ AAS62-61-P20 } & Porphyritic & 1 & 98.6 & -5.2 & 0.8 & -11.1 & 1.3 & -8.4 & 1.3 \\
\hline & & 2 & 89.9 & -26.9 & 0.9 & -32.9 & 1.2 & -18.9 & 1.3 \\
\hline & & 3 & 97.3 & -34.8 & 1.0 & -40.0 & 1.3 & -21.9 & 1.4 \\
\hline & & 4 & 76.4 & 30.9 & 0.8 & 9.2 & 1.3 & -6.8 & 1.3 \\
\hline & & 5 & 80.4 & 28.3 & 0.9 & 9.1 & 1.2 & -5.6 & 1.3 \\
\hline \multirow[t]{4}{*}{ AAS-38-204-P33 } & Porphyritic & 1 & 94.4 & -11.9 & 1.2 & -12.4 & 0.8 & -6.2 & 1.1 \\
\hline & & 2 & 89.4 & -6.2 & 1.2 & -7.9 & 0.9 & -4.7 & 1.1 \\
\hline & & 3 & 85.2 & 3.3 & 1.2 & -1.3 & 0.9 & -3.0 & 1.1 \\
\hline & & 4 & 75.3 & 8.0 & 1.2 & 2.0 & 0.8 & -2.2 & 1.0 \\
\hline \multirow[t]{2}{*}{ AAS62-32-P20 } & Porphyritic & 1 & 97.4 & -0.2 & 0.7 & -4.8 & 0.8 & -4.7 & 0.9 \\
\hline & & 2 & 93.8 & -4.6 & 0.6 & -7.8 & 0.8 & -5.4 & 0.8 \\
\hline \multirow[t]{2}{*}{ AAS62-61-P22 } & Relict bearing & 1 & 98.6 & -6.5 & 0.9 & -11.2 & 1.3 & -7.8 & 1.4 \\
\hline & & 2 & 98.7 & -3.3 & 0.9 & -10.7 & 1.3 & -8.9 & 1.4 \\
\hline \multirow[t]{2}{*}{ AAS-38-188-P43 } & Relict bearing & 1 & 98.8 & -5.1 & 1.1 & -6.9 & 1.6 & -4.2 & 1.7 \\
\hline & & 2 & 98.9 & -2.2 & 1.1 & -5.6 & 1.6 & -4.5 & 1.7 \\
\hline \multirow[t]{2}{*}{ AAS-62-51-P8 } & Porphyritic & 1 & 98.7 & -7.2 & 0.6 & -9.8 & 0.9 & -6.0 & 0.9 \\
\hline & & 2 & 99.1 & -6.3 & 0.7 & -8.6 & 0.9 & -5.3 & 1.0 \\
\hline \multirow[t]{3}{*}{ AAS-38-188-P15 } & Porphyritic & 1 & 98.0 & 4.9 & 1.0 & -0.9 & 1.6 & -3.4 & 1.7 \\
\hline & & 2 & 94.2 & 2.7 & 1.1 & -0.7 & 1.6 & -2.2 & 1.7 \\
\hline & & 3 & 83.2 & -9.3 & 1.0 & -12.1 & 1.6 & -7.3 & 1.7 \\
\hline \multirow[t]{3}{*}{ AAS62-40-P40 } & Porphyritic & 1 & 99.4 & -4.7 & 0.8 & -6.2 & 0.7 & -3.7 & 0.8 \\
\hline & & 2 & 99.3 & -4.6 & 0.6 & -5.4 & 0.7 & -3.0 & 0 \\
\hline & & 3 & 98.8 & -4.4 & 0.8 & -6.4 & 0.7 & -4.1 & 0.8 \\
\hline AAS62-40-P107 & Porphyritic & 1 & 98.2 & -7.3 & 0.8 & -9.1 & 0.8 & -5.3 & 0.9 \\
\hline AAS62-40-P108 & Porphyritic & 1 & 98.9 & -8.0 & 0.6 & -8.7 & 0.7 & -4.5 & 0.8 \\
\hline
\end{tabular}



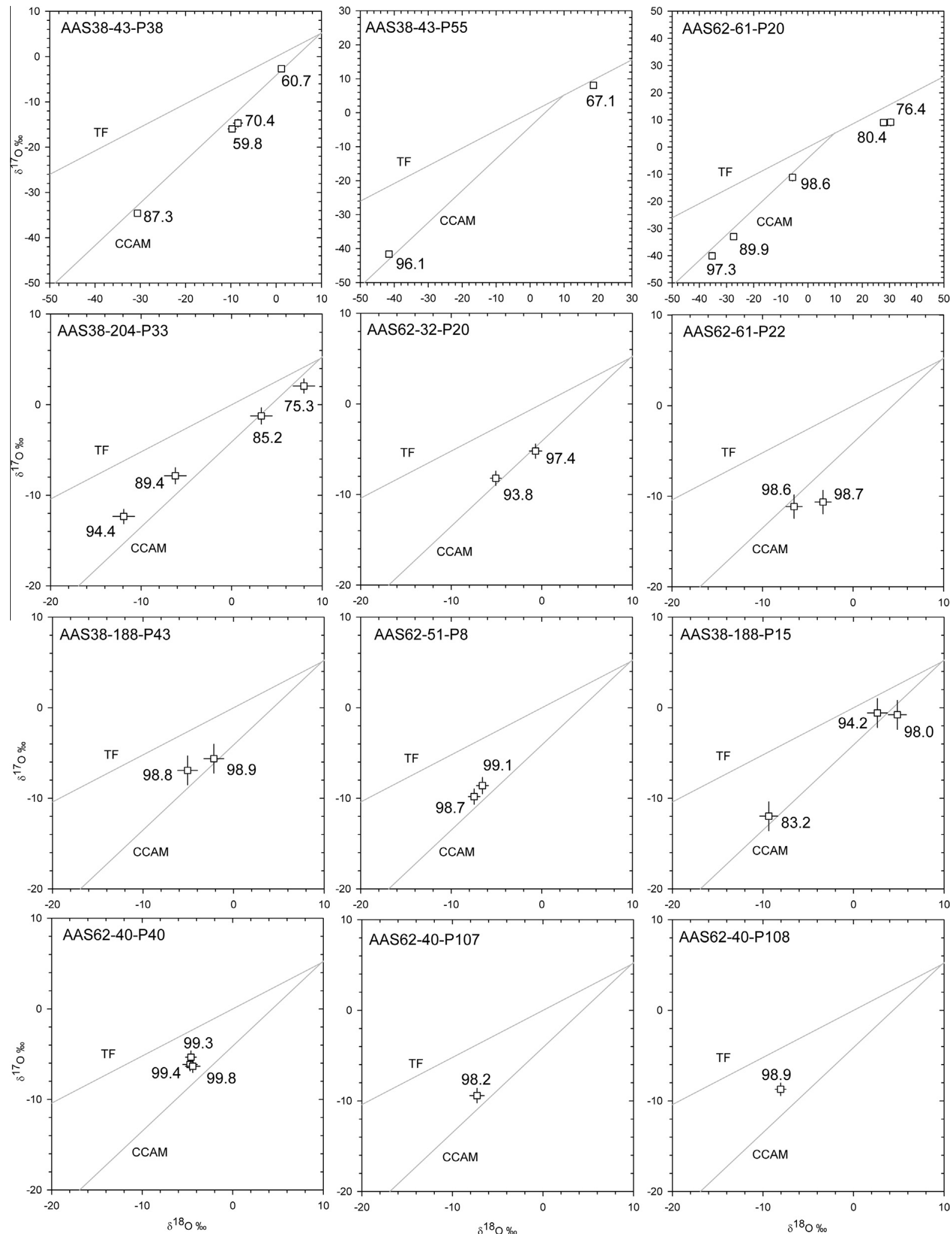

Fig. 4. Oxygen three isotope plots of relict olivine grains from cosmic spherules: BSE images of the cosmic spherules and the regions of analyses are shown in Figs. 1 and 2. The terrestrial fractionation (TF) and carbonaceous chondrite anhydrous mineral (CCAM) lines have slopes of $\sim 0.52$ and $\sim 0.94$, respectively (Clayton, 1993). All error bars are $2 \sigma$. The number in parentheses is the forsterite content (\%) of olivine. 

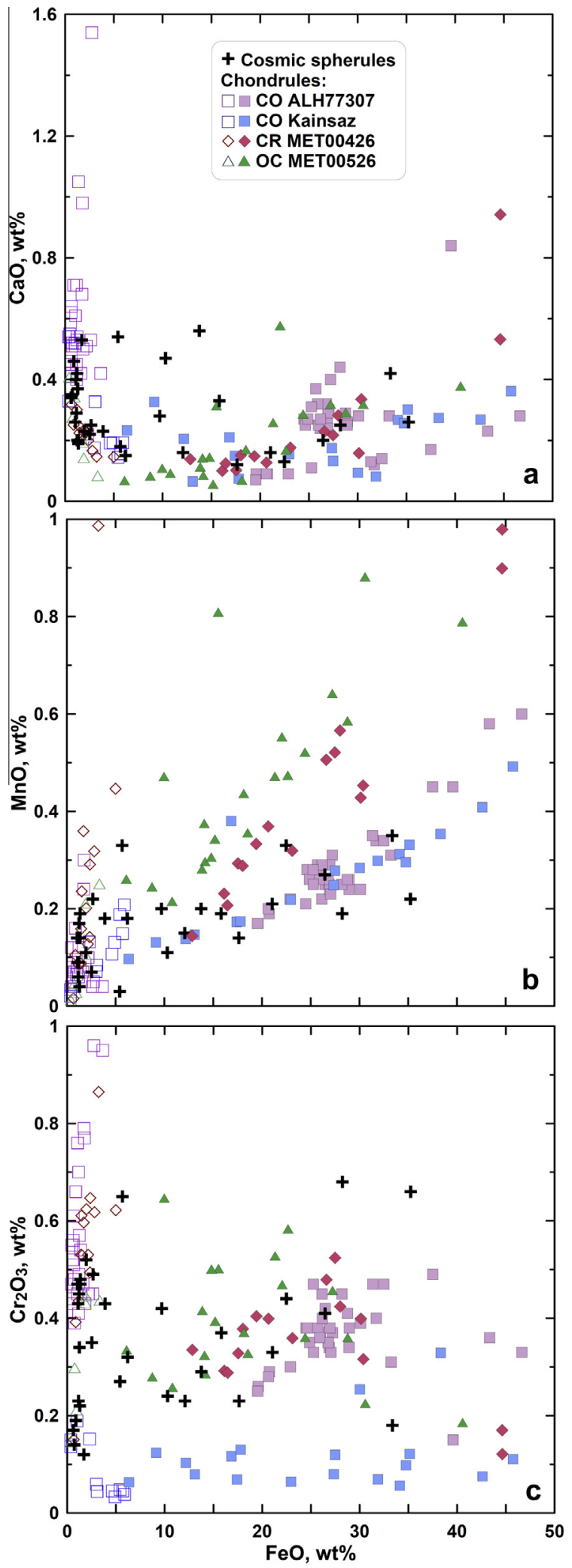

chondrites (e.g., Brearley and Jones, 1998). NiO contents in many olivine grains of spherules are $<0.2 \mathrm{wt} \%$, although contents up to $1.3 \mathrm{wt} \% \mathrm{NiO}$ were measured in the more $\mathrm{FeO}$-rich grains. Ni rich olivines are a consequence of heating during atmospheric entry (Michel-Lévy and Bourot-Denise, 1992; Steele, 1992; Beckerling and Bischoff, 1995; Gounelle et al., 2005; Cordier et al., 2011b). In addition to similar ranges, the distributions of minor elements relative to $\mathrm{FeO}$ are very similar to trends found in chondrules from unequilibrated chondrites, with forsteritic compositions $(\mathrm{FeO}<5 \mathrm{wt} \%)$ similar to olivine compositions in type I chondrules, and more FeO-rich compositions $(\mathrm{FeO}>20 \mathrm{wt} \%)$ similar to olivine compositions in type II chondrules (Fig. 5). In the plot of $\mathrm{CaO}$ vs. FeO (Fig. 5a), most cosmic spherule compositions are similar to type I and II chondrules of both ordinary and carbonaceous chondrites. Trends in Mn vs. Fe in type II chondrules are different depending on chondrite group (Berlin et al., 2011). In the plot of $\mathrm{MnO}$ vs. FeO (Fig. 5b), compositions of FeO-rich olivine from cosmic spherules are most consistent with $\mathrm{CO}$ chondrites. Olivine from type II chondrules in $\mathrm{CM}$ and $\mathrm{CV}$ chondrites shows the same trend as $\mathrm{CO}$ chondrites: Hanowski and Brearley (2001), Hewins et al. (2011), Jones (2012). Olivine compositions of cosmic spherules are also similar to those of chondrules in the plot of $\mathrm{Cr}_{2} \mathrm{O}_{3}$ vs. $\mathrm{FeO}$ (Fig. 5c). The $\mathrm{CO}$ chondrite, Kainsaz (petrologic subtype 3.2), has undergone mild metamorphism resulting in loss of $\mathrm{Cr}$ from FeO-rich olivine (e.g., Grossman and Brearley, 2005). Similarities in the $\mathrm{Cr}_{2} \mathrm{O}_{3}$ vs. FeO distribution between cosmic spherules and chondrules in $\mathrm{OC}$ MET00526 (subtype 3.05) and the primitive CO chondrite, ALH77307 (subtype 3.0), indicate that spherules have not undergone mild metamorphism on a parent body.

These observations support an origin for cosmic spherules relict grains from fragments of carbonaceous chondrite chondrules (Steele, 1992; Genge et al., 2005, 2008; Gounelle et al., 2005; Cordier et al., 2011a,b; Van Ginneken et al., 2012; Imae et al., 2013). This interpretation is consistent with the conclusions of previous studies based on chemical compositions, which have shown that the majority of 140

Fig. 5. Minor element $\left(\mathrm{CaO}, \mathrm{MnO}\right.$ and $\left.\mathrm{Cr}_{2} \mathrm{O}_{3}\right)$ contents of relict olivines from cosmic spherules where oxygen isotopes were analyzed, compared with olivine compositions in chondrules from unequilibrated ordinary and carbonaceous chondrites. Data for ALH77307 from Jones (1992) and Brearley and Jones (1998); remaining chondrule data from Berlin (2009) and Berlin et al. (2011). Open symbols are olivine compositions in type I chondrules, and closed symbols are olivine compositions in type II chondrules. Olivine in Kainsaz type I chondrules is slightly more FeO-rich than olivine in ALHA77307 type I chondrules, because Kainsaz has undergone mild metamorphism. Low $\mathrm{Cr}_{2} \mathrm{O}_{3}$ content in olivine in Kainsaz is also attributable to mild metamorphism (Grossman and Brearley, 2005). 


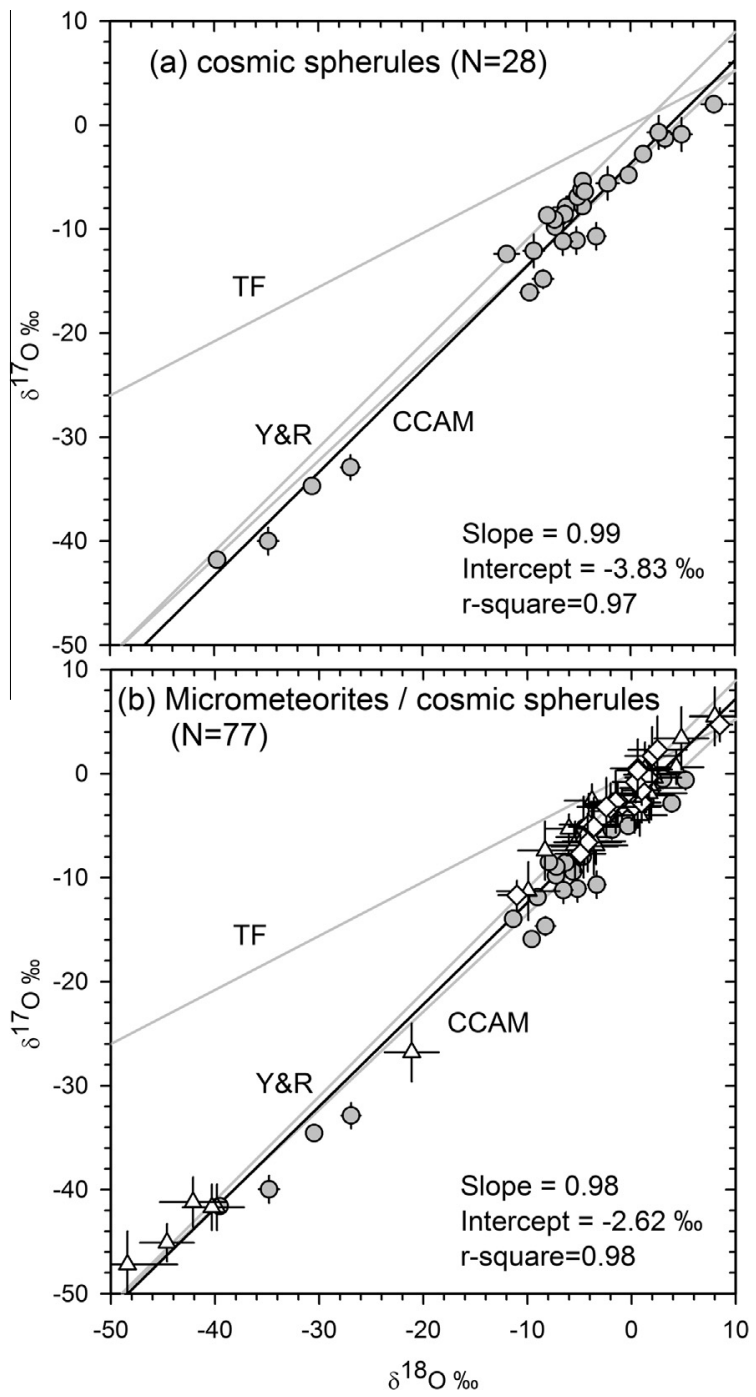

Fig. 6. (a) Oxygen three isotope plot for relict olivine grains $(N=28)$ in cosmic spherules from this study, excluding those having $\delta^{18} \mathrm{O}>10 \%$. "TF", "Y\&R" and "CCAM" represent the terrestrial mass fractionation line $\left(\delta^{17} \mathrm{O}=0.52 \times \delta^{18} \mathrm{O}\right)$, Young and Russell line $\left(\delta^{17} \mathrm{O}=1.00 \times \delta^{18} \mathrm{O}-1.04\right.$; from Young and Russell, 1998) and Carbonaceous Chondrite Anhydrous Mineral line $\left(\delta^{17} \mathrm{O}=0.94 \times \delta^{18} \mathrm{O}-4.1\right.$; Clayton et al., 1977). The dark line is a linear regression through data from the current work, which has the parameters given on the plot. All error bars are $2 \sigma$. (b) Oxygen isotopic compositions of all individual olivine and pyroxene grains in cosmic spherules including data of the present study (filled circles), Engrand et al. (1999) (triangles) and Gounelle et al. (2005) (diamonds). The dark line is a linear regression for all compiled data $(N=77)$ which has the parameters given on the plot. All error bars are $2 \sigma$.

relict olivine grains in micrometeorites from Antarctica and Greenland collections represent type I and type II chondrules of carbonaceous chondrites rather than ordinary chondrites (Beckerling and Bischoff, 1995; Engrand et al., 1999).
In addition to chemical relationships, oxygen isotope data of the olivine grains in cosmic spherules show a strong relationship to olivine grains in chondrules of carbonaceous chondrites. Fig. 6a shows a compilation of data from this study, and Fig. 6b shows a compilation including all previously published oxygen isotope data from relict grains of micrometeorites/cosmic spherules analyzed using ion microprobe (Engrand et al., 1999; Gounelle et al., 2005). Regression lines through all micrometeorites/cosmic spherules data in both Fig. $6 \mathrm{a}$ and $\mathrm{b}$ are very similar to the CCAM line (relict olivines in cosmic spherules AAS38-43-P55 and AAS62-61-P20 that have $\delta^{18} \mathrm{O}$ values $>20 \%$ are excluded from Fig. 6). Oxygen isotopic compositions of bulk chondrules from different carbonaceous chondrites also lie close to the CCAM line, as shown in Fig. 7, and have a range of $\delta^{18} \mathrm{O}$ values similar to that of the micrometeorites/cosmic spherules (Clayton, 1993; Weisberg et al., 1993; Rowe et al., 1994; Jones et al., 2004; Jones, 2012 and references therein; Jones et al., 2014a). Bulk chondrule data from the least metamorphosed ordinary chondrites such as Semarkona (LL3.0), Bishunpur (LL3.1) and Krymka (LL3.1) plot above the TF line, and there are not many olivine grains from micrometeorites/ cosmic spherules with such compositions in this region. Similarly, most cosmic spherule analyses do not overlap bulk oxygen isotopic compositions of chondrules from enstatite chondrites, which plot on the TF line. Many individual analyses from the micrometeorites overlap bulk chondrule compositions of carbonaceous $\mathrm{CV}, \mathrm{CK}, \mathrm{CR}$ and $\mathrm{CM}$ chondrites. It appears that the majority of the relict olivines in micrometeorites/cosmic spherules originated from chondrules of carbonaceous rather than ordinary and enstatite chondrites (Engrand et al., 1999). However, the bulk oxygen isotope study on large cosmic spherules by Suavet et al. (2010) has shown that $\sim 30 \%$ of them are related to ordinary chondrites.

Olivine grains in chondrules from carbonaceous chondrites such as Acfer 094 (Ushikubo et al., 2012), CO Yamato 81020 (Tenner et al., 2013), CR MET 00426 and QUE 99177 (Tenner et al., 2015), and CV3 Allende (Rudraswami et al., 2011b) show correlations between $\Delta^{17} \mathrm{O}$ and $\mathrm{Mg \#} \mathrm{(Fig.} \mathrm{8).} \mathrm{Grains} \mathrm{with} \mathrm{high} \mathrm{Mg \#} \mathrm{(>95)} \mathrm{in}$ Acfer $094, \mathrm{CO}$, and $\mathrm{CV}$ chondrites have $\Delta^{17} \mathrm{O}$ values close to $-5 \%$, while those with $\mathrm{Mg \#}<96$ in the same chondrites have values of $\Delta^{17} \mathrm{O} \sim 2 \%$ suggesting a bimodal distribution of oxygen isotope reservoirs in the chondrule forming region of the solar nebula (Rudraswami et al., 2011b; Ushikubo et al., 2012). An additional reservoir of $\Delta^{17} \mathrm{O}$ at $0 \%$ was also suggested (Rudraswami et al., 2011b). The ${ }^{16} \mathrm{O}$-rich group with high $\mathrm{Mg \#}$ and $\Delta^{17} \mathrm{O}$ at $-5 \%$ is typically associated with type I chondrules, while those with $\mathrm{Mg \#}<96$ and $\Delta^{17} \mathrm{O}$ at $-2 \%$ are associated with both type I and II chondrules (Ushikubo et al., 2012). In contrast, type I chondrules in CR chondrites have Mg\# $>95$ clustered at $-2 \%$ suggesting that they formed in a different environment compared to other carbonaceous chondrites (Tenner et al., 2015). Olivine grains with $\mathrm{Mg \#}>95$ of Comet Wild 2 particles are also grouped at $-2 \%$ and those 


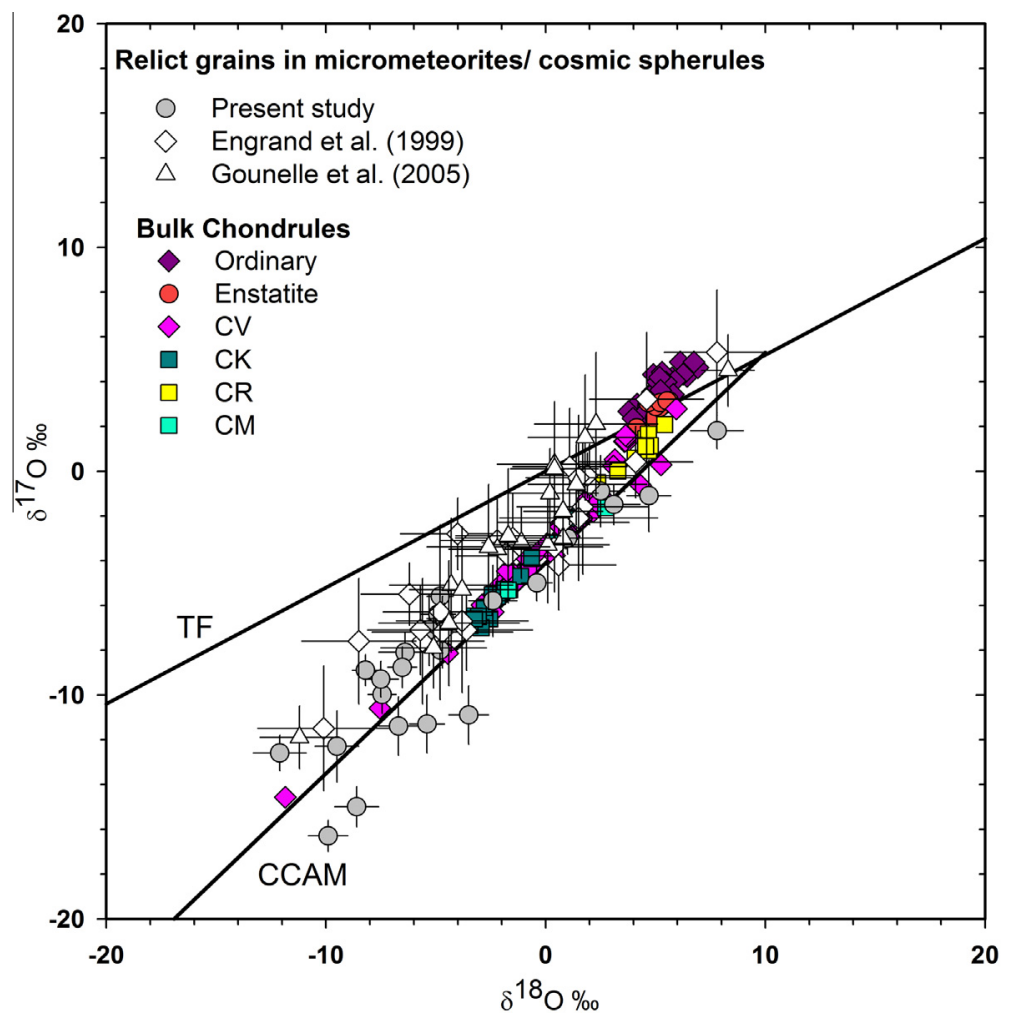

Fig. 7. Oxygen isotopic compositions for bulk chondrules in various carbonaceous and ordinary chondrites (Clayton et al., 1983, 1991; Clayton and Mayeda, 1985, 1999; Mayeda et al., 1988; Rubin et al., 1990; Weisberg et al., 1993; Rowe et al., 1994; Bridges et al., 1998; Jones et al., 2000, 2014a; Jones, 2012) are shown along with cosmic spherules data from the present study, Engrand et al. (1999) and Gounelle et al. (2005). The data for bulk chondrules are from least metamorphosed samples as discussed in Jones (2012). The solid lines are the TF and CCAM lines. All error bars of the micrometeorite/cosmic spherules data are $2 \sigma$.

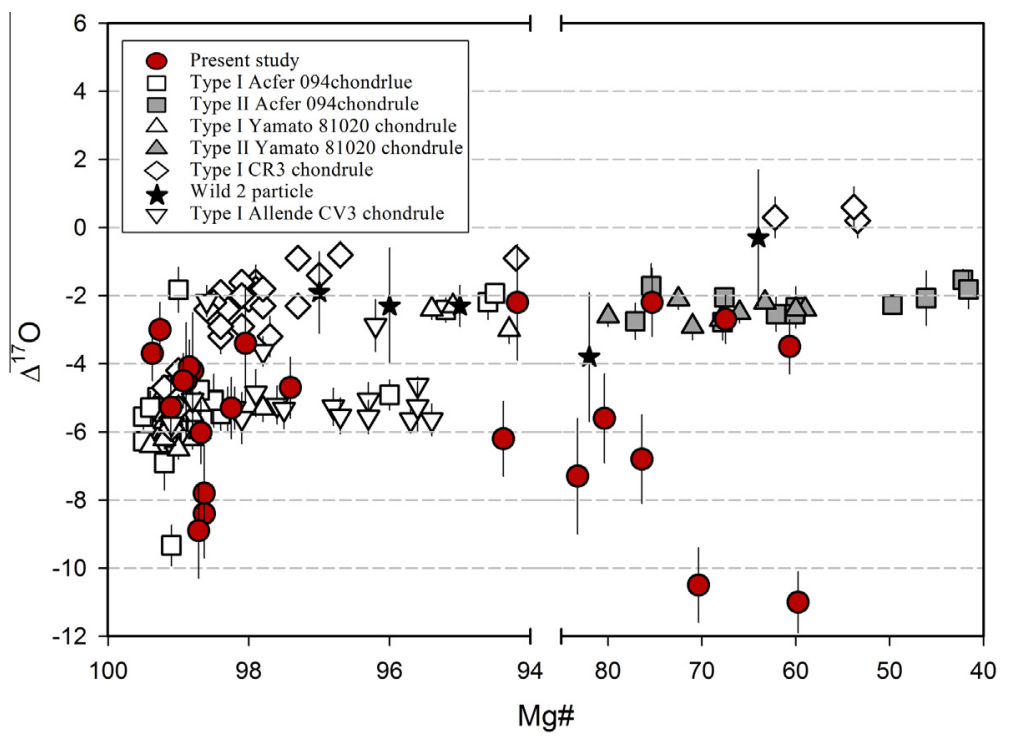

Fig. 8. The $\Delta^{17} \mathrm{O}$ values versus $\mathrm{Mg} \#$ of olivine of cosmic spherules. The data are compared with those from olivine of type I (FeO-poor) and II (FeO-rich) chondrules in Acfer 094 (Ushikubo et al., 2012) and Yamato 81020 (Tenner et al., 2013), type I chondrules of CR3 chondrite MET 00426 and QUE 99177 (Tenner et al., 2015), Type I chondrules of Allende CV3 (Rudraswami et al., 2011b) and comet Wild 2 particles (Nakashima et al., 2012). 
with $\mathrm{Mg \#}<80$ are scattered close to $-2 \%$ indicating a closer relation to $\mathrm{CR}$ chondrites than to $\mathrm{CO}, \mathrm{CV}$, and Acfer 094 (Nakashima et al., 2012). Comparison of the relict olivine with $\mathrm{Mg \#}>95$ from the present study shows that these grains have the same range as type I chondrules from $\mathrm{CO}$, $\mathrm{CV}$ and Acfer 094 carbonaceous chondrites, while few of the olivine grains with $\mathrm{Mg \#} 60$ to 75 match the type II chondrules from these groups. There are some olivines having $\Delta^{17} \mathrm{O}$ belonging to the type I chondrule group, but $\mathrm{Mg \#}$ similar to those found in type II groups (Fig. 8). The heating of the cosmic spherules during atmospheric entry may have resulted in disturbance of the $\mathrm{Mg \#}$ by changing the $\mathrm{Fe}-\mathrm{Mg}$ content, while retaining the original oxygen isotope composition due to its slow diffusion rate (Gerard and Jaoul, 1989; Cole and Chakraborty, 2001; Chakraborty, 2010).

\section{2. ${ }^{18} \mathrm{O}$-rich olivine grains and possible terrestrial contributions to oxygen isotopic compositions}

In two of the cosmic spherules (AAS38-43-P55 and AAS62-61-P20), $\delta^{18} \mathrm{O}$ values measured on some olivine grains are much higher than the majority of grains (Fig. 4; Table 3). The two olivine grains measured in AAS38-43-P55 have $\delta^{18} \mathrm{O}$ values of $+20.6 \%$ and $-39.5 \%$. We analyzed five olivine grains in AAS62-61-P20: three have negative $\delta^{18} \mathrm{O}$ values $(-5.2 \%,-26.9 \%,-34.8 \%$ o $)$, and two have high positive values, $+28.3 \%$ and $+30.9 \%$. The large positive $\delta^{18} \mathrm{O}$ values from AAS38-43-P55 and AAS62-61-P20 fall close to the TF line, at even higher $\delta^{18} \mathrm{O}$ values than those of hydrous minerals in CI chondrites, which range up to $+20 \%$ (Rowe et al., 1994). The olivine grains with high $\delta^{18} \mathrm{O}$ values are all relatively $\mathrm{FeO}-$ rich $\left(\mathrm{Fo}_{67}\right.$ in AAS38-43-P55, $\mathrm{Fo}_{76}$ and $\mathrm{Fo}_{80}$ in AAS62-61-P20) compared with olivine grains that have negative $\delta^{18} \mathrm{O}$ values in the same cosmic spherules $\left(\mathrm{Fo}_{96}\right.$ in $\mathrm{P} 55$ and $\mathrm{FO}_{90-99}$ in AAS62-61-P20). Highly negative $\Delta^{17} \mathrm{O}$ values in low $\mathrm{FeO}$ olivine grains and relatively less negative $\Delta^{17} \mathrm{O}$ in high $\mathrm{FeO}$ olivine grains are commonly found in many porphyritic chondrules (Jones et al., 2000; Wasson et al., 2004; Ushikubo et al., 2012; Tenner et al., 2013). It has been suggested that $\mathrm{FeO}$-rich grains may have exchanged oxygen with a ${ }^{16} \mathrm{O}$ depleted nebular reservoir during the formation of chondrules or multiple partial melting events (Wasson and Rubin, 2003). However, for the two spherules discussed here, oxygen isotopic compositions of the FeO-rich grains lie on or close to the CCAM line. Hence, it does not seem likely that this mechanism of nebular gas exchange is responsible for formation of the grains with high $\delta^{18} \mathrm{O}$ values in cosmic spherules AAS38-43-P55 and AAS62-61-P20. We consider two possible mechanisms for interpreting the high $\delta^{18} \mathrm{O}$ values: exchange of oxygen with terrestrial atmosphere upon atmospheric entry, and alteration processes on the parent body of the micrometeorite material.

During atmospheric entry, particles undergo varying degrees of heating depending on the angle of entry and velocity. Heating can result in mass loss via volatilization, which can result in isotopic fractionation. Also, heating can result in exchange of oxygen isotopic composition with the atmosphere (Engrand et al., 1999). The high $\delta^{18} \mathrm{O}$ values of olivine in spherules AAS38-43-P55 and AAS62-61-P20 may suggest that melting and isotopic exchange has occurred. However, the chemical compositions of the ${ }^{18} \mathrm{O}$-rich olivine grains in these spherules are similar to other grains that have isotopic compositions close to the CCAM line, which suggests that chemical compositions of the grains may not have been modified significantly. Spherule AAS38-43-P55 has a fine-grained, vesicular texture that indicates that it was melted during atmospheric entry, although AAS62-61-P20 has a porphyritic texture that does not obviously suggest the same process. Oxygen isotopic compositions of the grains with high $\delta^{18} \mathrm{O}$ are very similar to the oxygen isotope composition of tropospheric $\mathrm{O}_{2}, \delta^{18} \mathrm{O}=23.5 \%, \delta^{17} \mathrm{O}=11.8 \%$ (Thiemens et al., 1995), suggesting that these grains record isotopic exchange. However, the observed spread in $\delta^{18} \mathrm{O}$ within each spherule rules out complete isotopic mixing with atmospheric oxygen. Grains in the same spherules with oxygen isotopic compositions that lie along the CCAM line can be interpreted as relicts that survived the effects of atmospheric entry. In contrast, the origin of the high $\delta^{18} \mathrm{O}$ composition in some olivine is not very clear. To produce an increase in $\delta^{18} \mathrm{O}$, the $10 \mu \mathrm{m}$ grain with forsterite composition $98.5 \%$ can achieve $60 \%$ isotopic exchange with atmosphere within $40 \mathrm{~s}$ at $1500 \mathrm{~K}$; in the present study most of the grains are greater than $20 \mu \mathrm{m}$ (Engrand et al., 2005). The high values of $\delta^{18} \mathrm{O}$ in AAS38-43-P55 and AAS62-61-P20 may be the result of atmospheric oxygen isotope exchange during entry. The grains with high $\delta^{18} \mathrm{O}$ may have crystallized from a melted portion of the chondrule, during cooling. Accordingly, these grains have high $\mathrm{NiO}$ contents, $0.6-1.3 \mathrm{wt} \%$ NiO (Table 2) which could result from oxidation of metal during the atmospheric melting event.

An alternative explanation for grains with high $\delta^{18} \mathrm{O}$ in the cosmic spherules is that these grains became more depleted in ${ }^{16} \mathrm{O}$ as a result of aqueous alteration in the parent body (Rowe et al., 1994; Leshin et al., 1997). For CI and $\mathrm{CM}$ chondrites, aqueous alteration results in mass-dependent fractionation, and for CI chondrites, compositions of secondary hydrous phases spread along the TF line. Because the olivines analyzed in the present study with high $\delta^{18} \mathrm{O}$ value are anhydrous, we consider it unlikely that the high $\delta^{18} \mathrm{O}$ values are due to aqueous alteration. We favor an explanation that the high $\delta^{18} \mathrm{O}$ values represent atmospheric exchange.

Another potential terrestrial source of isotopic oxygen exchange for cosmic spherules is seawater, during the residence time in the ocean. Seawater has $\delta^{18} \mathrm{O}$ between $0 \%$ and $+1 \%$, and any isotopic exchange would drive oxygen isotope compositions toward this value (Clayton et al., 1986). An oxygen isotope study on basalt recovered from deep sea drilling has shown that oceanic rock exposed to cold seawater for millions of years differed in $\delta^{18} \mathrm{O}$ by less than $2 \%$ from its primary unaltered value (Muehlenbachs and Clayton, 1976). Redistribution or equilibration of oxygen isotopes is a function of temperature and time. With depth in the ocean, the rate of exchange of oxygen decreases rapidly due to decrease in temperature (Cole et al., 1987). The present study of olivine in cosmic spherules includes 
analyses with low $\delta^{18} \mathrm{O}$, down to $-40 \%$, and most analyses spread close to the CCAM line and away from the TF line (Fig. 6). This observation implies that there is no significant contribution of seawater in changing the isotopic signature of the spherules that have terrestrial ages of $\sim 50,000$ years (Prasad et al., 2013).

\section{3. ${ }^{16} \mathrm{O}$-rich olivine grains}

Oxygen isotope analyses of AAS-38-43-P38, AAS-38-43-P55, AAS62-61-P20 reveal the presence of ${ }^{16} \mathrm{O}$-rich forsteritic grains, with $\Delta^{17} \mathrm{O}$ values $-22 \%$ to $-19 \%$ (Table 3), similar to those found in CAIs and AOAs (Clayton et al., 1977, 1986; Kimura et al., 1993; Hiyagon and Hashimoto, 1999; Krot et al., 2004b, 2005). ${ }^{16} \mathrm{O}$-rich olivine is also found in chondrules of Allende, Mokoia, Murchison, Yamato-86009, Acfer094 and other carbonaceous and ordinary chondrites (e.g., Hiyagon and Hashimoto, 1999; Jones et al., 2004; Rudraswami et al., 2011b; Ushikubo et al., 2012). Similarly, ${ }^{16}$ O-rich forsteritic grains have been reported from comet Wild 2 (McKeegan et al., 2006; Nakamura et al., 2008; Nakamura-Messenger et al., 2011; Nakashima et al., 2012) and stratospheric interplanetary dust particles (IDPs) (McKeegan, 1987).

For cosmic spherules AAS-38-43-P38 and AAS-62-61-P20, ${ }^{16} \mathrm{O}$-rich and ${ }^{16} \mathrm{O}$-poor grains are observed in the same spherules, with very small spatial separation of $<200 \mu \mathrm{m}$. This is the first observation of olivine grains having both ${ }^{16} \mathrm{O}$-rich and ${ }^{16} \mathrm{O}$-poor compositions within the same cosmic spherules. The coexistence of grains with very different $\Delta^{17} \mathrm{O}$ values along the CCAM line indicates incomplete oxygen isotope exchange, most likely during formation of the original cosmic spherules. Isotopic heterogeneity on this scale suggests that some grains preserve their original isotopic record. For particles with high porosity, heterogeneity during heating creates a thermal gradient which results in atmospheric oxygen exchange during entry only with some grains (Matrajt et al., 2006). The textures of cosmic spherules AAS-38-43-P38 and AAS-62-61-P20 resemble type II chondrules, in which larger FeO-rich olivine grains commonly have ${ }^{16} \mathrm{O}$-rich, forsteritic cores that are relict in the sense that they survived chondrule formation (Jones et al., 2000; Kunihiro et al., 2004; Ruzicka et al., 2007; Kita et al., 2008; Connolly and Huss, 2010; Ushikubo et al., 2012; Schrader et al., 2013; Tenner et al., 2013). Similar to chondrules, ${ }^{16}$ O-rich forsterite grains in these two cosmic spherules may have been mixed with ${ }^{16} \mathrm{O}$-poor material in fine-grained precursor material, prior to a melting event, or there may have been oxygen isotope exchange with the nebular gas when the precursor of these cosmic spherules were molten. We suggest that ${ }^{16} \mathrm{O}$-rich olivine in these cosmic spherules records primitive conditions in the solar nebula, similar to primary condensates that have oxygen isotopic compositions close to the Solar composition determined from the Genesis mission (McKeegan et al., 2011). Overall, it appears that ${ }^{16} \mathrm{O}$-rich olivines are similar to refractory inclusions or AOAs, and could have formed in the inner protoplanetary disk region at an early stage of the solar nebula, then later migrated to a ${ }^{16} \mathrm{O}$-poor chondrule-forming region, closer to the chondrite accretion region (Itoh and Yurimoto, 2003). Similar to chondrules, cosmic spherules are the products of high temperature events that resulted in partial melting and preservation of relict precursor grains. It appears that the original isotopic signatures of relict grains in cosmic spherules have not been disturbed subsequently, either as a result of heating or other alteration events on their parent bodies, atmospheric entry or during their residence time in seawater.

\subsection{Oxygen isotope distribution}

As discussed above, the majority of the relict olivines in cosmic spherules investigated here fall in the carbonaceous chondrite region of the oxygen three-isotope plot (Figs. 6 and 7). Bulk chondrule oxygen isotope compositions for various chondrite groups are shown for comparison with olivine grain analyses of cosmic spherules (Fig. 7). Chondrules from carbonaceous chondrites such as $\mathrm{CV}$, CK, CR and CM (Fig. 7) have compositions close to the CCAM line (e.g., Clayton et al., 1983; Rubin et al., 1990; Jones et al., 2004; Rudraswami et al., 2011b; Jones, 2012; Ushikubo et al., 2012). Oxygen isotope data for cosmic spherules mostly falls between the CCAM (Clayton et al., 1977) and Y\&R (Young and Russell, 1998) lines (Fig. 6). The present study (excluding a few points having higher $\delta^{18} \mathrm{O}>20 \%$ ) has a best fit slope of 0.99 and an intercept of $-3.8 \%$ (Fig. 6). By comparison the ion microprobe data from the current study combined with previous investigations on olivine grains of micrometeorites using ion microprobe (Engrand et al., 1999; Gounelle et al., 2005) has a similar slope (0.98) and an intercept of $-2.6 \%$. These slopes are similar to the Primitive Chondrule Minerals (PCM) line (slope of $\sim 0.99$ ) defined by Ushikubo et al. (2012) and to those found for chondrules of CV3 chondrites including Mokoia and Allende (Jones et al., 2004, 2014a; Rudraswami et al., 2011b). The best fit lines for bulk chondrule oxygen isotopes for $\mathrm{CV}, \mathrm{CK}, \mathrm{CR}$ and $\mathrm{CM}$ chondrites have slopes of $0.99,0.99,0.99$ and 0.82 , respectively (see summary in Jones (2012); along with additional data from Rowe et al. (1994) and Clayton and Mayeda (1999) for chondrules of Essebi CM and Karoonda CK chondrites, respectively, Jones et al. (2014a) for CV chondrites Allende, Mokoia, Vigarano and Leoville, and Jones et al. (2014b) for primitive CR chondrite EET 92042). The slopes for chondrules from $\mathrm{CV}, \mathrm{CK}$ and primitive $\mathrm{CR}$ chondrites resemble micrometeorite/cosmic spherule results. Chondrules from CM chondrites have undergone aqueous alteration. The spread of the cosmic spherules olivine grains lies very close to bulk chondrules of $\mathrm{CV}, \mathrm{CK}, \mathrm{CR}$ and $\mathrm{CM}$ chondrites, making it difficult to distinguish a relationship with any particular chondrite group. No data are available for chondrules from $\mathrm{CO}$ chondrites, due to their smaller size; however, in situ analyses of individual grains show that they fall approximately on the CCAM line (Clayton et al., 1983, 1991; Clayton and Mayeda, 1985, 1999; Mayeda et al., 1988; Rubin et al., 1990; Weisberg et al., 1993; Rowe et al., 1994; Bridges et al., 1998; Jones et al., 2000; Tenner et al., 2013). Isolated olivine grains from CI chondrites lie on the CCAM line but are ${ }^{16} \mathrm{O}$-poor compared 

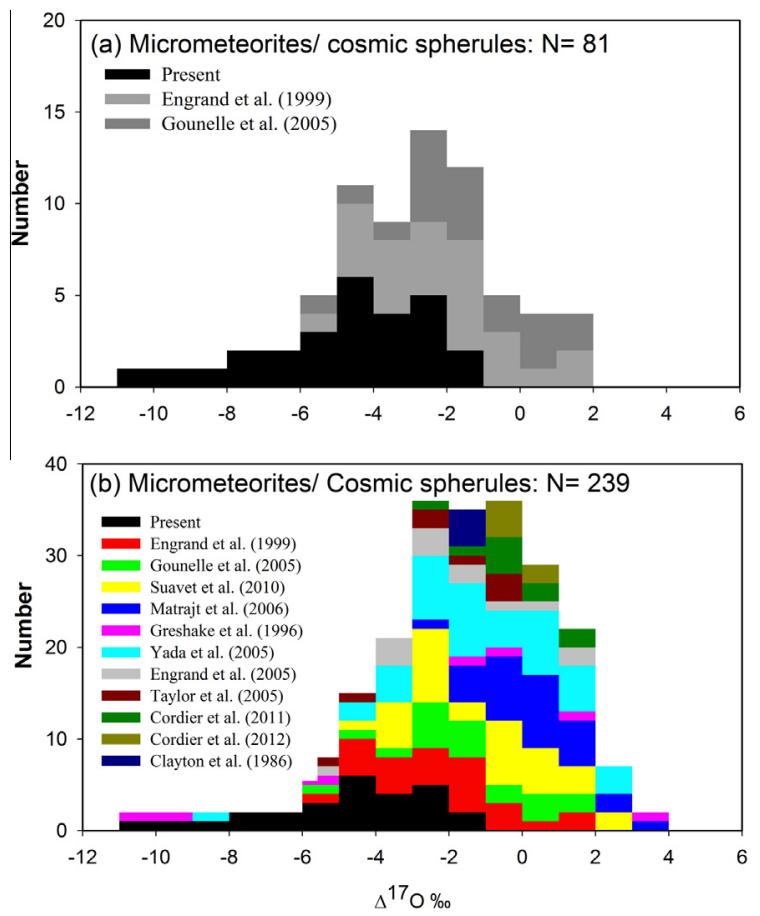

Fig. 9. (a) Histogram plot of $\Delta^{17} \mathrm{O}$ compiled for in situ analyses of individual relict grains in micrometeorites/cosmic spherules for all analyses in the present study and those by Engrand et al. (1999) and Gounelle et al. (2005). (b) Histogram plot of $\Delta^{17} \mathrm{O}$ of individual relict grains, bulk analyses and in situ spot analyses of IDPs and micrometeorites/cosmic spherules (Clayton et al., 1986; Greshake et al., 1996; Engrand et al., 1999, 2005; Gounelle et al., 2005; Taylor et al., 2005; Yada et al., 2005; Matrajt et al., 2006; Suavet et al., 2010; Cordier et al., 2011a, 2012).

to other carbonaceous chondrites (Leshin et al., 1997). The $\delta^{17,18} \mathrm{O}$ values of olivine in cosmic spherules are within the range of chondrules from carbonaceous chondrites, and very few data fall in the range defined for bulk ordinary and enstatite chondrites.

Nearly $\sim 80 \%$ of the micrometeorites with relict grains from this study and the work of Engrand et al. (1999) and Gounelle et al. (2005) have $\Delta^{17} \mathrm{O}$ ranging from $-8 \%$ to $+2 \%$ (Fig. 9a). Also the compilation of the oxygen isotope analyses from in situ and bulk analyses of IDPs (interplanetary dust particles) and all types of micrometeorites/cosmic spherules (melted, partially melted and unmelted) have a $\Delta^{17} \mathrm{O}$ spread within the same range (Clayton et al., 1986; Greshake et al., 1996; Engrand et al., 1999, 2005; Gounelle et al., 2005; Taylor et al., 2005; Yada et al., 2005; Matrajt et al., 2006; Suavet et al., 2010; Cordier et al., 2011a, 2012; Cordier and Folco, 2014), showing that IDPs and micrometeorites/cosmic spherules represent comparable populations of interplanetary material (Fig. 9b). The bulk oxygen isotope compositions of unmelted micrometeorites (e.g., Matrajt et al., 2006) and those of cosmic spherules (Cordier and Folco, 2014) have higher $\delta^{17,18} \mathrm{O}$. Furthermore, oxygen isotope data from comet Wild 2 particle fragments are similar to those of chondrules in carbonaceous chondrites and fall close to the CCAM line (McKeegan et al., 2006;
Nakamura et al., 2008; Nakashima et al., 2012). Hence, close relationships can be inferred between oxygen isotopes of micrometeorites, cosmic spherules, chondrules, IDPs, and comet Wild 2 fragments, suggesting their formation in similar oxygen isotope reservoirs before becoming incorporated into different parent bodies (Engrand et al., 1999, 2005). Chondrules that accreted into parent bodies close to their formation zones became constituents of chondritic parent bodies, whereas chondrules that were transported outward, possibly as a result of gas flow in the midplane, became constituents of comets in the Kuiper Belt region (Ciesla, 2007). Micrometeorites and cosmic spherules could thus have either have an asteroidal or a cometary origin.

Dust arising from the asteroid belt and ejecta from comets provides a wide variety of solar system materials that we sample on Earth as micrometeorites. Both dust populations are broadly chondritic, but there is also a minor achondritic contribution to micrometeorite material (Taylor et al., 2007; Gounelle et al., 2009; Badjukov et al., 2010; Cordier et al., 2011a, 2012). One of the reasons that micrometeorites are dominated by chondritic material could be that micrometeorites are sourced largely from C-type asteroids or comets. These bodies have properties such as low density, high porosity and low material strength, as a result of which they are fragmented easily during collisions and would end up as dust size particles (Consolmagno et al., 2008). Among meteorites, the bulk densities of $\mathrm{CI}$ and $\mathrm{CM}$ chondrites are the lowest due to high porosity $\left(\sim 2 \mathrm{gm} / \mathrm{cm}^{3}\right.$, Britt et al., 2002; Consolmagno et al., 2008). The other carbonaceous chondrites do not differ significantly from ordinary chondrites ( $\sim 2.9$ to $3.5 \mathrm{gm} / \mathrm{cm}^{3}$ : Britt et al., 2002). The porosity of C-type asteroids, $>20 \%$, is high compared with the ordinary chondrites $(<10 \%)$ (Consolmagno et al., 2008). Although many carbonaceous chondrites do not show high porosity and are similar to ordinary chondrites (average porosity 11-16\%: Britt et al., 2002), samples in our meteorite collections may not be representative of all the C-type asteroids: our sampling may be biased toward material with low porosity that is likely to maintain its integrity and not suffer catastrophic disruption. Infrared astronomical satellite observations suggest that release of dust in the asteroid belt is a by-product of disruptive collisions, and most of the dust contribution is from C-type asteroid or asteroid clusters (Nesvorný et al., 2003). This contrasts with the fact that ordinary chondrites are the most common meteorite falls. Comets also play an important role in the contribution of interplanetary dust; thus, their contribution to the flux of micrometeorites cannot be ignored (Cremonese et al., 2012). The Stardust spacecraft returned cometary samples that show oxygen isotope compositions are similar to chondrules and refractory inclusions in carbonaceous chondrites (McKeegan et al., 2006; Nakamura et al., 2008; Nakashima et al., 2012). The oxygen isotope data of comets and carbonaceous chondrites probably overlap as particle formation and migration has taken place from inner solar nebula region to the Kuiper belt (Ciesla, 2007; Aléon et al., 2009; Ushikubo et al., 2012). Because chemical and isotopic compositions of micrometeorites overlap with both carbonaceous chondrites and comets, it is not possible at 
present to accurately distinguish between these two probable sources.

\section{CONCLUSIONS}

Relict olivine grains in the cosmic spherules in the present study have sampled asteroidal or cometary parent bodies. They show chemical and oxygen isotope compositions analogous to chondrules from various types of carbonaceous chondrites and some of them have textures that strongly resemble fragments of individual chondrules. Their chemical and isotopic compositions demonstrate that they have largely escaped atmospheric entry processing. The majority of oxygen isotope compositions measured in the relict olivine grains of cosmic spherules are clustered along the CCAM line, at $\Delta^{17} \mathrm{O}$ ranging from $-5 \%$ to $0 \%$, which indicates formation in a ${ }^{16} \mathrm{O}$-poor reservoir similar to that recorded by chondrules from carbonaceous chondrites. In addition, ${ }^{16} \mathrm{O}$-rich compositions of some olivine grains indicate that the conditions of their formation may be similar to that experienced by CAIs and AOAs. The association of ${ }^{16} \mathrm{O}$-rich forsteritic olivine and ${ }^{16} \mathrm{O}$-poor $\mathrm{FeO}$-rich olivine in the same cosmic spherules is analogous to relict forsterite grains and their $\mathrm{FeO}$-rich overgrowths observed in type II chondrules in carbonaceous and ordinary chondrites.

In two of the cosmic spherules we studied, some olivine grains show high $\delta^{18} \mathrm{O}$ values, up to $+30 \%$, which are higher than have been observed in any meteorite group. The high value could be attributed to isotopic exchange with stratospheric oxygen during atmospheric entry. A compilation of all the relict grains analyzed in cosmic spherules to date has a slope of $\sim 0.99$, similar to compiled data for chondrules from $\mathrm{CV}, \mathrm{CK}$ and $\mathrm{CR}$ chondrites, indicating a strong relationship between these extraterrestrial materials.

\section{ACKNOWLEDGMENTS}

N.G.R. and M.S.P. are also grateful to the GEOSINKS (Council of Scientific and Industrial Research XII Plan) and PLANEX (Physical Research Laboratory, Ahmedabad) project, under which this work has been carried out. N.G.R. would also thank Indo-US Science and Technology Forum (IUSSTF) for providing the fellowship and support to carry out the research. We thank Carole Cordier and two anonymous reviewers for their constructive comments and suggestions. R.J. acknowledges financial support from NASA Cosmochemistry grant NNX12AH61G. The laboratory assistance of Vijay Khedekar and Samena Balagar is gratefully acknowledged. We would like to thank Gary Huss are Sasha Krot for their support. This is NIO contribution No. 5753.

\section{REFERENCES}

Aléon J., Engrand C., Leshin L. A. and McKeegan K. D. (2009) Oxygen isotopic composition of chondritic interplanetary dust particles: a genetic link between carbonaceous chondrites and comets. Geochim. Cosmochim. Acta 73, 4558-4575.

Badjukov D. D., Brandstatter F., Raitala J. and Kurat G. (2010) Basaltic micrometeorites from the Novaya Zemlya glacier. Meteorit. Planet. Sci. 45, 1502-1512.
Beckerling W. and Bischoff A. (1995) Occurrence and composition of relict minerals in micrometeorites from Greenland and Antarctica-implications for their origins. Planet. Space Sci. 43, 435-449.

Berlin J. (2009) Mineralogy and bulk chemistry of chondrules and matrix in petrologic type 3 chondrites: implications for early solar system processes. $\mathrm{Ph}$. D. thesis, University of New Mexico.

Berlin J., Jones R. H. and Brearley A. J. (2011) Fe-Mn systematics of type IIA chondrules in unequilibrated $\mathrm{CO}, \mathrm{CR}$, and ordinary chondrites. Meteorit. Planet. Sci. 46, 513-533.

Brearley A. J. and Jones R. H. (1998) Chondritic meteoritesPapike, J. J. (ed.). Planetary Materials. Reviews in Mineralogy 36, 3-13-398.

Bridges J. C., Franchi I. A., Hutchison R., Sexton A. S. and Pillinger C. T. (1998) Correlated mineralogy, chemical compositions, oxygen isotopic compositions and size of chondrules. Earth Planet. Sci. Lett. 155, 183-196.

Britt D. T., Yeomans D., Housen K. and Consolmagno G. (2002) Asteroid density, porosity, and structure. In Asteroids III (ed. , Jr.W. F. Bottke et al.). Univ. of Arizona, Tucson, pp. 485-500.

Brownlee D. E. (1985) Cosmic dust: collection and research. Annu. Rev. Earth Planet. Sci. 13, 147-173.

Brownlee D. E., Bates B. and Schramm L. (1997) The elemental composition of stony cosmic spherules. Meteorit. Planet. Sci. 32, 157-175.

Chakraborty S. (2010) Diffusion coefficients in olivine, wadsleyite, and ringwoodite. In Reviews in Mineralogy and Geochemistry, 72 (eds. Y. Zhang and D. J. Cherniak). Mineralogical Society of America, Washington, DC, pp. 603-639.

Ciesla F. J. (2007) Outward transport of high-temperature materials around the midplane of the solar nebula. Science 318, 613615.

Clayton R. N. (1993) Oxygen isotopes in meteorites. Annu. Rev. Earth Planet. Sci. 21, 115-149.

Clayton R. N. and Mayeda T. K. (1985) Oxygen isotopes in chondrules from enstatite chondrites: possible identification of a major nebular reservoir. 16th Lunar Planet. Sci. Conf. pp. $142-143$ (abstr.).

Clayton R. N. and Mayeda T. K. (1999) Oxygen isotope studies of carbonaceous chondrites. Geochim. Cosmochim. Acta 63, 20892104.

Clayton R. N., Onuma N., Grossman L. and Mayeda T. K. (1977) Distribution of pre-solar component in Allende and other carbonaceous chondrites. Earth Planet. Sci. Lett. 34, 209-224.

Clayton R. N., Onuma N., Ikeda Y., Mayeda T. K., Hutcheon I. D., Olsen E. J. and Molini-Velsko C. (1983) Oxygen isotopic compositions of chondrules in Allende and ordinary chondrites. In Chondrules and Their Origins (ed. E. A. King). Lunar and Planetary Institute, Houston, pp. 37-43.

Clayton R. N., Mayeda T. M. and Brownlee D. E. (1986) Oxygen isotopes in deep-sea spherules. Earth Planet. Sci. Lett. 79, 235240.

Clayton R. N., Mayeda T. K., Goswami J. N. and Olsen E. J. (1991) Oxygen isotope studies of ordinary chondrites. Geochim. Cosmochim. Acta 55, 2317-2337.

Cole D. R., Mottl M. J. and Ohmoto H. (1987) Isotopic exchange in mineral-fluid systems. II. Oxygen and hydrogen isotopic investigation of the experimental basalt-seawater system. Geochim. Cosmochim. Acta 63, 2623-2636.

Cole D. R. and Chakraborty S. (2001) Rates and mechanisms of isotope exchange. In Reviews in Mineralogy and Geochemistry, 43 (eds. J. W. Valley and D. R. Cole). Mineralogical Society of America, Washington, DC, pp. 83-223.

Connolly, Jr., H. C. and Huss G. R. (2010) Compositional evolution of the protoplanetary disk: oxygen isotopes of type-II 
chondrules from CR2 chondrites. Geochim. Cosmochim. Acta 74, 2473-2483.

Consolmagno G. J., Britt D. T. and Macke R. J. (2008) The significance of meteorite density and porosity. Chem. Erde 68, $1-29$.

Cordier C. and Folco L. (2014) Oxygen isotopes in cosmic spherules and the composition of the near Earth interplanetary dust complex. Geochim. Cosmochim. Acta 146, 18-26.

Cordier C., Folco L., Suavet C., Sonzogni C. and Rochette P. (2011a) Major, trace element and oxygen isotope study of glass cosmic spherules of chondritic composition: the record of their source material and atmospheric entry heating. Geochim. Cosmochim. Acta 75, 5203-5218.

Cordier C., van Ginneken M. and Folco L. (2011b) Nickel abundance in stony cosmic spherules: constraining precursor material and formation mechanisms. Meteorit. Planet. Sci. 46, $1110-1132$.

Cordier C., Suavet C., Folco L., Rochette P. and Sonzogni C. (2012) HED-like cosmic spherules from the Transantarctic Mountains, Antarctica: major and trace element abundances and oxygen isotopic compositions. Geochim. Cosmochim. Acta $77,515-529$.

Cremonese G., Borin P., Martellato E., Marzari F. and Bruno M. (2012) New calibration of the micrometeoroid flux on earth. Astrophys. J. Lett. 749, L40.

Engrand C., McKeegan K. D. and Leshin L. A. (1999) Oxygen isotopic compositions of individual minerals in Antarctic micrometeorites: further links to carbonaceous chondrites. Geochim. Cosmochim. Acta 63, 2623-2636.

Engrand C., McKeegan K. D., Leshin L. A., Herzog G. F., Schnabel C., Nyquist L. E. and Brownlee D. E. (2005) Isotopic compositions of oxygen, iron, chromium, and nickel in cosmic spherules: toward a better comprehension of atmospheric entry heating effects. Geochim. Cosmochim. Acta 69, 5365-5385.

Fagan T. J., Krot A. N., Keil K. and Yurimoto H. (2004) Oxygen isotopic evolution of amoeboid olivine aggregates in the reduced CV3 chondrites Efremovka, Vigarano and Leoville. Geochim. Cosmochim. Acta 68, 2591-2611.

Genge M. J., Gileski A. and Grady M. M. (2005) Chondrules in Antarctic micrometeorites. Meteorit. Planet. Sci. 40, 225-238.

Genge M. J., Engrand C., Gounelle M. and Taylor S. (2008) The classification of micrometeorites. Meteorit. Planet. Sci. 43, 497 515.

Gerard O. and Jaoul O. (1989) Oxygen diffusion in San-Carlos olivine. J. Geophys. Res. 94, 4119-4128.

Gounelle M., Engrand C., Maurette M., Kurat G., McKeegan K. D. and Brandstätter F. (2005) Small Antarctic micrometeorites: a mineralogical and in situ oxygen isotopic study. Meteorit. Planet. Sci. 40, 917-932.

Gounelle M., Chaussidon M., Morbidelli A., Barrat J. A., Engrand C., Zolensky M. E. and McKeegan K. D. (2009) A unique basaltic micrometeorite expands the inventory of solar system planetary crusts. Proc. Natl. Acad. Sci. USA 106, 6904-6909.

Greshake A., Bischoff A. and Hopee P. (1996) Mineralogy, chemistry, and oxygen isotopes of refractory inclusions from stratospheric interplanetary dust particles and micrometeorites. Meteorit. Planet. Sci. 31, 739-748.

Greshake A., Klock W., Arndt P., Maetz M., Flynn G. J., Bajt S. and Bischoff A. (1998) Heating experiments simulating atmospheric entry heating of micrometeorites: clues to their parent body sources. Meteorit. Planet. Sci. 33, 267-290.

Grossman J. N. and Brearley A. J. (2005) The onset of metamorphism in ordinary and carbonaceous chondrites. Meteorit. Planet. Sci. 40, 87-122.

Hanowski N. and Brearley A. J. (2001) Aqueous alteration of chondrules in the CM carbonaceous chondrite, Allan Hills
81002: implications for parent body alteration. Geochim. Cosmochim. Acta 65, 495-518.

Hewins R. H., Zanda B. and Bourot-Denise M. (2011) The formation of type II chondrules in CM chondrites: the view from Paris. 42nd Lunar Planet. Sci. Conf. \#1914 (abstr.).

Hiyagon H. and Hashimoto A. (1999) ${ }^{16} \mathrm{O}$ excesses in olivine inclusions in Yamato-86009 and Murchison chondrites and their relation to CAIs. Science 283, 828-831.

Imae N., Taylor S. and Iwata N. (2013) Micrometeorite precursors: clues from the mineralogy and petrology of their relict minerals. Geochim. Cosmochim. Acta 100, 113-157.

Imai H. and Yurimoto H. (2003) Oxygen isotopic distribution in an amoeboid olivine aggregate from the allende $\mathrm{CV}$ chondrite: primary and secondary processes. Geochim. Cosmochim. Acta 67, 765-772.

Itoh S. and Yurimoto H. (2003) Contemporaneous formation of chondrules and refractory inclusions in the early Solar System. Nature 423, 728-731.

Jones R. H. (1992) On the relationship between isolated and chondrule olivine grains in the carbonaceous chondrite, ALH A77307. Geochim. Cosmochim. Acta 56, 467-482.

Jones R. H. (2012) Petrographic constraints on the diversity of chondrule reservoirs in the protoplanetary disk. Meteorit. Planet. Sci. 47, 1176-1190.

Jones R. H., Saxton J. M., Lyon I. C. and Turner G. (2000) Oxygen isotopes in chondrule olivine and isolated olivine grains from the CO3 chondrite Allan Hills A77307. Meteorit. Planet. Sci. 35, 849-857.

Jones R. H., Leshin L. A., Guan Y., Sharp Z. D., Durakiewicz T. and Schilk A. J. (2004) Oxygen isotope heterogeneity in chondrules from the Mokoia CV3 carbonaceous chondrite. Geochim. Cosmochim. Acta 68, 3423-3438.

Jones R. H., Rudraswami N. G., Ziegler K. (2014a) Primary oxygen isotope distribution in chondrules and refractory inclusions from $\mathrm{CV}$ carbonaceous chondrites. 45th Lunar Planet. Sci. Conf. \#1512 (abstr.).

Jones R. H., Ziegler K., Windler G. (2014b) A primitive oxygen isotope reservoir for chondrules from CR chondrites. 77 th Annual Meeting of the Meteoritical Society, \#5165.

Kimura M., El Goresy A., Palme H. and Zinner E. (1993) Ca-Alrich inclusions in the unique chondrite ALH 85085 - petrology, chemistry, and isotopic compositions. Geochim. Cosmochim. Acta 57, 2329-2359.

Kita N. T., Kimura M., Ushikubo T., Valley J. W. and Nyquist L. E. (2008) Oxygen isotope systematics of chondrules from the least equilibrated $\mathrm{H}$ chondrite. 39th Lunar Planet. Sci. Conf. CD-ROM. \#2059.

Kita N. T., Nagahara H., Tachibana S., Tomomura S., Spicuzza M. J., Fournelle J. H. and Valley J. W. (2010) High precision SIMS oxygen three isotope study of chondrules in LL3 chondrites: role of ambient gas during chondrule formation. Geochim. Cosmochim. Acta 74, 6610-6635.

Krot A. N., Fagan T. J., Keil K., McKeegan K. D., Sahijpal S., Hutcheon I. D., Petaev M. I. and Yurimoto H. (2004a) Ca, Alrich inclusions, amoeboid olivine aggregates, and Al-rich chondrules from the unique carbonaceous chondrite Acfer 094: I. Mineralogy and petrology. Geochim. Cosmochim. Acta 68, 2167-2184.

Krot A. N., Petaev M. I., Russell S. S., Itoh S., Fagan T. J., Yurimoto H., Chizmadia L., Weisberg M. K., Komatsu M., Ulyanov A. A. and Keil K. (2004b) Amoeboid olivine aggregates and related objects in carbonaceous chondrites: records of nebular and asteroid processes. Chem. Erde 64, 185239.

Krot A. N., Fagan T. J., Nagashima K., Petaev M. I. and Yurimoto H. (2005) Origin of low-Ca pyroxene in amoeboid 
olivine aggregates; evidence from oxygen isotopic compositions. Geochim. Cosmochim. Acta 69, 1873-1881.

Kunihiro T., Rubin A. E., McKeegan K. and Wasson J. T. (2004) Oxygen-isotopic compositions of relict and host grains in chondrules in the Yamato 81020 CO3.0 chondrite. Geochim. Cosmochim. Acta 68, 3599-3606.

Kurat G., Koeberl C., Presper T., Brandstatter F. and Maurette M. (1994) Petrology and geochemistry of Antarctic micrometeorites. Geochim. Cosmochim. Acta 58, 3879-3904.

Leshin L. A., Rubin A. E. and McKeegan K. D. (1997) The oxygen isotopic composition of olivine and pyroxene from CI chondrites. Geochim. Cosmochim. Acta 61, 835-845.

Lodders K. and Fegley, Jr., B. (1998) The Planetary Scientist's Companion. Oxford University Press, New York.

Love S. G. and Brownlee D. E. (1993) A direct measurement of the terrestrial mass accretion rate of cosmic dust. Science 262, 550553.

Matrajt G., Guan Y., Leshin L., Taylor S., Genge M., Joswiak D. and Brownlee D. (2006) Oxygen isotope measurements of individual unmelted Antarctic micrometeorites. Geochim. Cosmochim. Acta 70, 4007-4018.

Mayeda T. K., Clayton R. N., Kring D. A. and Davis A. M. (1988) Oxygen, silicon and magnesium isotopes in Ningqiang chondrules. Meteoritics 23, 288.

McKeegan K. D. (1987) Oxygen isotopic abundances in refractory stratospheric dust particles: proof of extraterrestrial origin. Science 237, 1468-1471.

McKeegan K. D. and Leshin L. A. (2001) Stable isotope variations in extraterrestrial materials. In Stable Isotope Geochemistry, 43 (eds. J. W. Valley and D. R. Cole), pp. 279-318. Reviews in Mineralogy and Geochemistry. Mineralogical Society of America, Washington, DC.

McKeegan K. D., Aléon J., Bradley J., Brownlee D., Busemann H., Butterworth A., Chaussidon M., Fallon S., Floss C., Gilmour J., Gounelle M., Graham G., Guan Y., Heck P. R., Hoppe P., Hutcheon I. D., Huth J., Ishii H., Ito M., Jacobsen S. B., Kearsley A., Leshin L. A., Liu M.-C., Lyon I., Marhas K., Marty B., Matrajt G., Meibom A., Messenger S., Mostefaoui S., Mukhopadhyay S., Nakamura-Messenger K., Nittler L., Palma R., Pepin R. O., Papanastassiou D. A., Robert F., Schlutter D., Snead C. J., Stadermann F. J., Stroud R., Tsou P., Westphal A., Young E. D., Ziegler K., Zimmermann L. and Zinner E. (2006) Isotopic compositions of cometary matter returned by Stardust. Science 314, 1724-1728.

McKeegan K. D., Kallio A. P. A., Heber V. S., Jarzebinski G., Mao P. H., Coath C. D., Kunihiro T., Wiens R. C., Nordholt J. E., Moses, Jr., R. W., Reisenfeld D. B., Jurewicz A. J. G. and Burnett D. S. (2011) The oxygen isotopic composition of the Sun inferred from captured Solar Wind. Science 332, 15281532.

Michel-Lévy M. C. and Bourot-Denise M. (1992) Mineral compositions in Antarctic and Greenland micrometeorites. Meteoritics 27, 73-80.

Muehlenbachs K. and Clayton R. N. (1976) Oxygen isotope composition of the Oceanic crust and its bearing on seawater. $J$. Geophys. Res. 81, 4365-4369.

Nakamura T., Noguchi T., Tsuchiyama A., Ushikubo T., Kita N. T., Valley J. W., Zolensky M. E., Kakazu Y., Sakamoto K., Mashio E., Uesugi K. and Nakano T. (2008) Chondrule-like objects in short-period comet 81P/Wild 2. Science 321, 1664 1667.

Nakamura-Messenger K., Keller L. P., Clemett S. J., Messenger S. and Ito M. (2011) Nanometerscale anatomy of entire Stardust tracks. Meteorit. Planet. Sci. 46, 1033-1051.

Nakashima D., Ushikubo T., Joswiak D. J., Brownlee D. E., Matrajt G., Weisberg M. K., Zolensky M. E. and Kita N. T.
(2012) Oxygen isotopes in crystalline silicates of comet Wild 2: a comparison of oxygen isotope systematics between Wild 2 particles and chondritic materials. Earth Planet. Sci. Lett. 357358, 355-365.

Nesvorný D., Bottke W. F., Levison H. F. and Dones L. (2003) Recent origin of the solar system dust bands. Astrophys. J. 591, 486-497.

Prasad M. S., Rudraswami N. G. and Panda D. K. (2013) Micrometeorite flux on earth during the last $\sim 50,000$ years. $J$. Geophys. Res. 118, 2381-2399.

Rowe M. W., Clayton R. N. and Mayeda T. K. (1994) Oxygen isotopes in separated components of CI and CM meteorites. Geochim. Cosmochim. Acta 58, 5341-5347.

Rubin A. E., Wasson J. T., Clayton R. N. and Mayeda T. K. (1990) Oxygen isotopes in chondrules and coarse-grained chondrule rims from the Allende meteorite. Earth Planet. Sci. Lett. 96, 247-255.

Rudraswami N. G., Parashar K. and Shyam Prasad. M. (2011a) Micrometer and nanometer size platinum group nuggets in micrometeorites from the deep sea sediments of Indian Ocean. Meteorit. Planet. Sci. 46, 470-491.

Rudraswami N. G., Ushikubo T., Nakashima D. and Kita N. T. (2011b) Oxygen isotope systematics of chondrules in Allende CV3 chondrite: high precision ion microprobe studies. Geochim. Cosmochim. Acta 75, 7596-7611.

Rudraswami N. G., Shyam Prasad. M., Babu E. V. S. S. K., Vijaya Kumar. T., Feng W. and Plane J. M. C. (2012) Fractionation and fragmentation of glass cosmic spherules during atmospheric entry. Geochim. Cosmochim. Acta 99, 110-127.

Ruzicka A., Hiyagon H., Hutson M. and Floss C. (2007) Relict olivine, chondrule recycling, and the evolution of nebular oxygen reservoirs. Earth Planet. Sci. Lett. 257, 274-289.

Schrader D. L., Connolly, Jr., H. C., Lauretta D. S., Nagashima K., Huss G. R., Davidson J. and Domanik K. J. (2013) The formation and alteration of the Renazzo-like carbonaceous chondrites II: linking O-isotope composition and oxidation state of chondrule olivine. Geochim. Cosmochim. Acta 101, 302327.

Steele A. (1992) Olivine in Antarctic micrometeorites: comparison with other extraterrestrial olivine. Geochim. Cosmochim. Acta 56, 2923-2929.

Suavet C., Alexandre A., Franchi I. A., Gattacceca J., Sonzogni C., Greenwood R. C., Folco L. and Rochette P. (2010) Identification of the parent bodies of micrometeorites with high-precision oxygen isotope ratios. Earth Planet. Sci. Lett. 293, 313-320.

Taylor S., Lever J. H. and Harvey R. P. (2000) Numbers, types and compositions of an unbiased collection of cosmic spherules. Meteorit. Planet. Sci. 35, 651-666.

Taylor S., Alexander C. M. O., Delaney J. S., Ma P., Herzog G. F. and Engrand C. (2005) Isotopic fractionation of iron, potassium, and oxygen in stony cosmic spherules: implications for heating histories and sources. Geochim. Cosmochim. Acta 69, $2647-2662$.

Taylor S., Herzog G. F. and Delaney J. S. (2007) Crumbs from the crust of Vesta: achondritic cosmic spherules from the South Pole water well. Meteorit. Planet. Sci. 42, 223-233.

Taylor S., Matrajt G. and Guan Y. (2012) Fine-grained precursors dominate the micrometeorite flux. Meteorit. Planet. Sci. 47, 550-564.

Tenner T. J., Ushikubo T., Kurahashi E., Nagahara H. and Kita N. T. (2013) Oxygen isotope systematics of chondrule phenocrysts from the CO3.0 chondrite Yamato 81020: evidence for two distinct oxygen isotope reservoirs. Geochim. Cosmochim. Acta 102, 226-245. 
Tenner T. J., Nakashima D., Ushikubo T., Kita N. T. and Weisberg M. K. (2015) Oxygen isotope ratios of FeO-poor chondrules in CR 3 chondrites: influence of dust enrichment and $\mathrm{H}_{2} \mathrm{O}$ during chondrule formation. Geochim. Cosmochim. Acta 148, 228-250.

Thiemens M. H., Jackson T., Zipf E. C., Erdman P. W. and Van Egmond C. (1995) Carbon dioxide and oxygen isotope anomalies in the mesosphere and stratosphere. Science 270, 969-972.

Ushikubo T., Kimura M., Kita N. T. and Valley J. W. (2012) Primordial oxygen isotope reservoirs of the Solar nebula recorded in chondrules in Acfer 094 carbonaceous chondrites. Geochim. Cosmochim. Acta 90, 242-264.

Van Ginneken M., Folco L., Cordier C. and Rochette P. (2012) Chondritic micrometeorites from the Transantarctic Mountains. Meteorit. Planet. Sci. 47, 228-247.

Wasson J. T. and Rubin A. E. (2003) Ubiquitous relict grains in type-II chondrules, narrow overgrowths, and chondrule cooling rates following the last melting event. Geochim. Cosmochim. Acta 67, 2239-2250.

Wasson J. T., Yurimoto H. and Russell S. S. (2001) ${ }^{16} \mathrm{O}$-rich melilite in $\mathrm{CO} 3.0$ chondrites: possible formation of common, ${ }^{16} \mathrm{O}$-poor melilite by aqueous alteration. Geochim. Cosmochim. Acta 65, 4539-4549.

Wasson J. T., Rubin A. E. and Yurimoto H. (2004) Evidence in CO3.0 chondrules for a drift in the O-isotopic composition of the solar nebula. Meteorit. Planet. Sci. 39, 1591-1598.
Weisberg M. K., Prinz M., Clayton R. N. and Mayeda T. K. (1993) The CR (Renazzo-type) carbonaceous chondrite group and its implications. Geochim. Cosmochim. Acta 57, 1567-1586.

Yada T., Nakamura T., Takaoka N., Noguchi T., Terada K., Yano H., Nakazawa T. and Kojima H. (2004) The global accretion rate of extraterrestrial materials in the last glacial period estimated from the abundance of micrometeorites in Antarctic glacier ice. Earth Planets Space 56, 67-79.

Yada T., Nakamura T., Noguchi T., Matsumoto N., Kusakabe M., Hiyagon H., Ushikubo T., Sugiura N., Kojima H. and Takaoka N. (2005) Oxygen isotopic and chemical compositions of cosmic spherules collected from the Antarctic ice sheet: implications for their precursor materials. Geochim. Cosmochim. Acta 69, 5789-5804.

Young E. D. and Russell S. S. (1998) Oxygen reservoirs in the early solar nebula inferred from an Allende CAI. Science 282, 452455.

Yurimoto H. and Wasson J. T. (2002) Extremely rapid cooling of a carbonaceous-chondrite chondrule containing very ${ }^{16} \mathrm{O}$-rich olivine and a ${ }^{26} \mathrm{Mg}$-excess. Geochim. Cosmochim. Acta 66, 4355-4363.

Yurimoto H., Ito M. and Nagasawa H. (1998) Oxygen isotope exchange between refractory inclusion in allende and solar nebula Gas. Science 282, 1874-1877.

Associate editor: Chris Herd 